\title{
Occurrence frequencies of IMF triggered and nontriggered substorms
}

\author{
Tung-Shin Hsu and Robert L. McPherron \\ Institute of Geophysics and Planetary Physics, University of California, Los Angeles, Los Angeles, California, USA
}

Received 12 April 2002; revised 6 January 2003; accepted 25 April 2003; published 29 July 2003.

[1] The occurrence of triggered and nontriggered substorm are examined in light of current interest in such issues as substorm identification, IMF $\mathrm{B}_{\mathrm{y}}$ variations, and potentially undetected small-scale solar wind perturbation. Global substorms are identified using a sudden, persistent decrease in the AL index. The onset of this global expansion is taken to be the time of the Pi 2 burst nearest in time to the beginning of the AL decrease. IMF triggers were identified both subjectively through visual scanning of the data and automatically with a computer algorithm. Both northward turnings of the IMF Bz and decreases in the amplitude of the By component were considered as possible triggers. Two different solar wind monitors were used in the investigation: IMP-8 in a circular orbit with a distance 12 35 Re to the Earth-Sun line and ISEE-2 in an elliptical orbit with a distance only $5 \sim 10$ Re to the Earth-Sun line. The IMP-8 results show that the triggering probability does not depend on the distance of the monitor from the Earth-Sun line in the range 12-35 Re. The ISEE dataset shows that closer than $12 \mathrm{Re}$ the triggering probability is the same as it is in the IMP- 8 data set. Thus there appears to be no dependence of triggering on the location of the monitor provided it is within $35 \mathrm{Re}$ of the Earth. We also demonstrate that including the By component does not significantly increase the probability of substorm triggering. Approximately $60 \%$ of all substorms appear to be triggered. Of the $40 \%$ for which we could not identify a trigger, $10 \%$ occurred while the IMF was northward. The data suggest that substorm onset is a consequence of an internal magnetospheric instability that is highly sensitive to changes in magnetospheric convection induced by a sudden change in the IMF, but that these changes are not always necessary. INDEX TERMS: 2784 Magnetospheric Physics: Solar wind/magnetosphere interactions; 2740 Magnetospheric Physics: Magnetospheric configuration and dynamics; 2788

Magnetospheric Physics: Storms and substorms; 2799 Magnetospheric Physics: General or miscellaneous; KEYWORDS: magnetospheric substorm, solar wind trigger, triggering probability

Citation: Hsu, T.-S., and R. L. McPherron, Occurrence frequencies of IMF triggered and nontriggered substorms, J. Geophys. Res., 108(A7), 1307, doi:10.1029/2002JA009442, 2003.

\section{Introduction}

[2] A prerequisite to understanding substorms is the formulation of a global description of the morphology of a substorm. This global picture should be consistent with phenomena observed within the ionosphere and within the magnetosphere. Also, it should be able to distinguish substorms from other types of geomagnetic disturbances. After almost 30 years of investigation this global picture is rather well defined.

[3] Another prerequisite is to determine whether substorms are the result of an internal instability of the magnetosphere or whether they are a response to changes in the solar wind or interplanetary magnetic field (IMF). Most substorm theories ascribe the cause of substorms to an internal instability [e.g., Coroniti, 1985; Hones et al., 1973; Lui et al., 1988; Roux et al., 1991]. However, there is a growing body of evidence that substorms can be triggered by the IMF [Blanchard et al., 2000; Caan et al., 1975,

Copyright 2003 by the American Geophysical Union. 0148-0227/03/2002JA009442\$09.00
1977; Lyons, 1996b; Lyons et al., 1997; Rostoker, 1983; Rostoker et al., 1983; Samson and Yeung, 1986]. The question of whether a substorm must always be triggered by IMF perturbations has become a controversial issue lately [Lyons, 1995].

[4] A statistical survey of the relative occurrence frequency of a variety of triggering disturbances was performed by McPherron et al. [1986]. In this study the authors concluded that substorm onsets were caused by a process internal to the magnetosphere, since about $30 \%$ of their events occurred when IMF $B_{z}$ was steadily southward. Horwitz [1985] also showed that substorm onsets could occur in the absence of major directional changes in the IMF $B_{z}$ and $\mathrm{B}_{\mathrm{y}}$. These observations are significant because they suggest that substorms can occur as the result of a purely internal magnetospheric instability.

[5] However, recently, Lyons [1995, 1996b] has challenged the results of McPherron et al. [1986]. He suggests that any process that may lead to a reduction of the magnetospheric electric field should be taken into account. This means IMF $\mathrm{B}_{\mathrm{z}}$ as well as IMF $\left|\mathrm{B}_{\mathrm{y}}\right|$ [Troshichev et al., 1986]. Lyons pointed out that two of the nontriggered 
substorm examples published by McPherron et al. [1986] may have been caused by IMF $\left|B_{y}\right|$. The first example is presented in Figure 1 and it shows that a clear reduction of IMF $\left|\mathrm{B}_{\mathrm{y}}\right|$ occurred at the time of the sudden perturbation of the $\mathrm{AL}$ index. For the second example in Figure 2 is not so easy to determine whether the IMF $\left|\mathrm{B}_{\mathrm{y}}\right|$ change was a trigger for this event. From such examples we conclude that the inclusion of IMF $\left|\mathrm{B}_{\mathrm{y}}\right|$ may actually change the occurrence frequency of triggered substorms. Although the works of Burch [1973], Caan et al. [1977], Dmitrieva and Sergeev [1983], and McPherron et al. [1986] showed that indeed there are examples of substorms that do not have obvious triggers in the solar wind, a statistical study that includes IMF $\left|\mathrm{B}_{\mathrm{y}}\right|$ as a possible trigger has not been performed.

[6] Another objection raised by Lyons [1996b] is the timing procedure used for determining expansion phase onsets. Both McPherron et al. [1986] and Horwitz [1985] relied on sharp perturbations in the $\mathrm{AL}$ or $\mathrm{AE}$ index to determine the onset time. It is well known that geomagnetic disturbances can be separated into two distinct types DP-1 and DP-2 [e.g., Nishida, 1968]. The DP-1 is associated with the substorm expansion electrojet and the DP-2 is due to large-scale convection within the magnetosphere [e.g., Iijima and Nagata, 1972; Yahnin et al., 1994]. While these two current systems are very different, both affect the $\mathrm{AL}$ index. The ambiguity of using AL to determine substorm
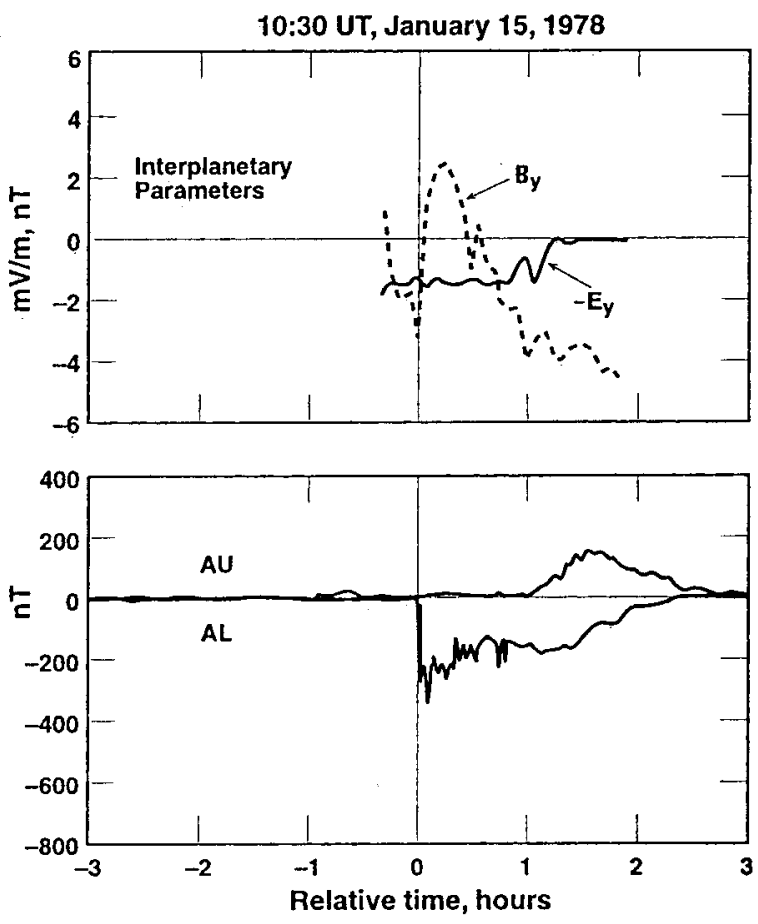

Figure 1. Event chosen by McPherron et al. [1986] as an example of stable IMF $B_{z}$ conditions at the time of expansion phase onset. $\mathrm{B}_{\mathrm{z}}$ is only shown where it is negative and is plotted as the negative of the y component of the interplanetary electric field. The IMF $\mathrm{B}_{\mathrm{y}}$ has been added to the original figure to illustrate the possibility that a change in $\mathrm{B}_{\mathrm{y}}$ might have triggered this event (reproduced from Lyons [1996b]).
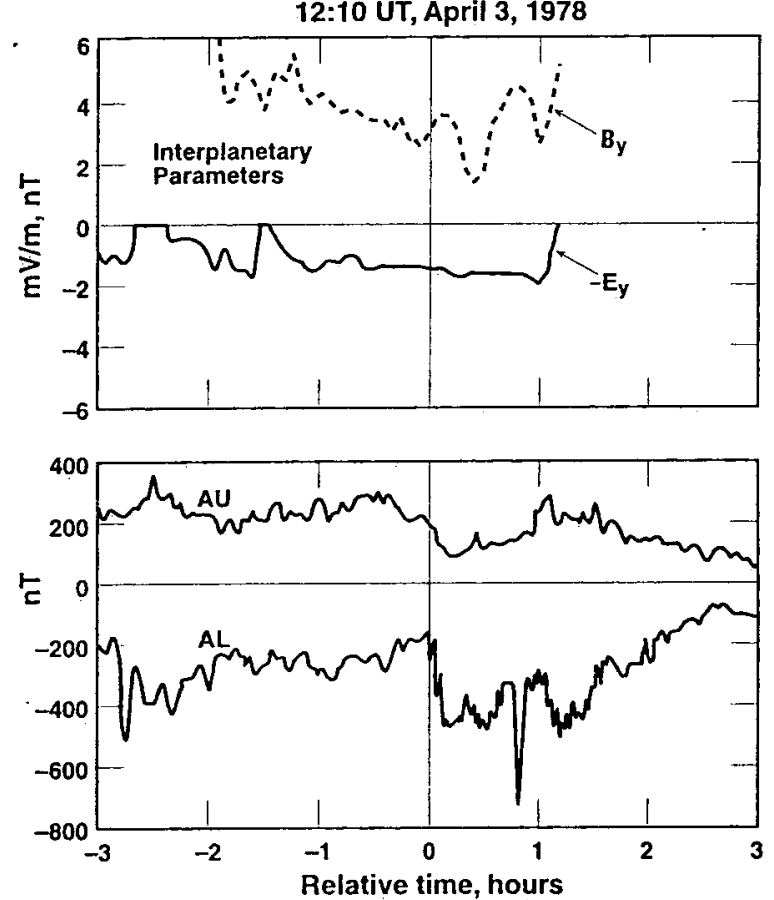

Figure 2. A second event chosen by McPherron et al. [1986] as a stable IMF $B_{z}$ at the onset of the expansion phase. The IMF $B_{y}$ was added later and does show a small variation at the time of the onset, but it is hard to tell if this is a significant change (reproduced from Lyons [1996b]).

onsets can be clearly seen in Figure 3. While AL shows a significant change in magnitude after $1200(\sim-1000 \mathrm{nT})$, the midlatitude stations do not show the positive perturbation in $\mathrm{H}$ characteristic of onset until 1600 UT. Thus it is possible that one could mistakenly identify intervals of enhanced convection as a substorm when only the $\mathrm{AL}$ index is used.

[7] Recently, investigators have used both case studies and statistical studies to address this issue. Henderson et al. [1996] used a much more comprehensive data set than previous investigators (with particle data from the Los Alamos energetic particle detectors at geosynchronous orbit, IMP-8 magnetometer and plasma data, Viking UV auroral image data, midlatitude Pi 2 pulsations, ground magnetometer data, and ISEE-1 magnetic field and energetic particle data) to investigate this problem. They found several events that appear to be substorms, even by the Lyons definition, for which there was no evidence of an IMF or solar wind trigger. Henderson et al. concluded that the model of Lyons [1995] could not successfully explain their observations.

[8] In rebuttal, Lyons et al. [1997] claimed that there is another parameter important in deciding whether a substorm has been triggered. This is the perpendicular distance $(\rho)$ from the Earth-Sun line to the solar wind monitor. They suggested that as the solar wind monitor approaches the Earth-Sun line, the probability of observing an apparent IMF trigger increases. Indeed, Lyons et al. [1997] used 20 substorm events to investigate this hypothesis and found 


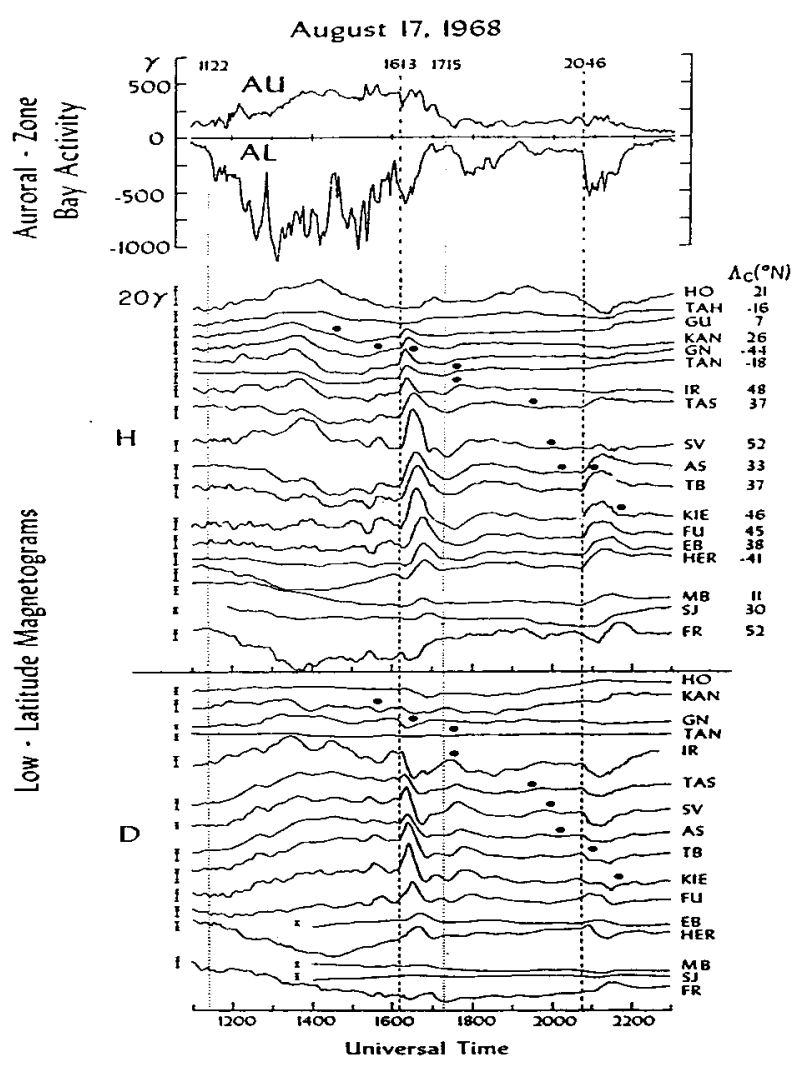

Figure 3. $\mathrm{AU}$ and $\mathrm{AL}$ indices, and $\mathrm{H}$ and $\mathrm{D}$ component midlatitude magnetograms for 17 August 1968. Heavy dots above each magnetogram indicate the UT of local magnetic midnight. Vertical lines indicate expansion phase onset (from Pytte et al. [1978]). Note the significant AE index for several hours preceding the substorm onset identified by midlatitude positive bay and Pi 2 pulsations. Such intervals are called "convection bays" and are thought to be different from substorms.

that the chance of observing triggered substorms was higher when the solar wind monitor was near the Earth-Sun line. This hypothesis that the magnetosphere is driven by smallscale solar wind structures that hit the subsolar magnetopause obviously introduces another variable in the problem of deciding whether substorm onset requires an external change or can occur spontaneously without a northward turning of the IMF.

[9] The work in this paper is motivated by our desire to determine whether all substorms are externally triggered. Here we use a statistical approach to address the questions of how frequently substorms appear to be triggered and how this frequency depends on position of the solar wind monitor. Several issues raised in recent studies will be examined: (1) Does the inclusion of IMF $\mathrm{B}_{\mathrm{y}}$ cause a significant change in the percentage of triggered events, and (2) does the occurrence frequency of apparently triggered substorms increase significantly as the monitor moves toward the Earth-Sun line?

[10] As discussed in preceding papers [Lyons, 1996a, 1996b], a substorm indicator other than AL should be chosen to avoid the problem of inaccurate onset times. Here we will use the procedure developed by Hsu and McPherron
[1996, 1998] that improves the AL onset time by finding the beginning of the Pi 2 closest in time to the AL onset. With this procedure we will examine how frequently a substorm expansion can be triggered by either IMF $B_{z}$ or $\left|B_{y}\right|$ perturbations. Then, we will examine whether the "smallscale structure hypothesis" is valid.

\section{Databases Used in Examining the Triggering Hypothesis}

[11] In this study we use two different databases to examine the triggering hypothesis. The first was obtained during spring of 1978 and 1979. The other was obtained during the fall of 1978 and 1979. Each of these datasets has its own advantages and disadvantages for the study.

\subsection{Substorm Onset Timing}

[12] A substorm onset timing procedure is proposed by Hsu and McPherron [1996, 1998, 2002]. This procedure scans the AL index from the auroral oval for any possible enhancement of auroral electrojet. Then high time resolution magnetometer data $(<3 \mathrm{~s})$ from either the AFGL [see Knecht, 1985; McPherron, 1992] or IGS [see Stuart, 1982] magnetometer chains were analyzed. The north components from the entire chain of stations were band pass filtered in the Pi 2 band $(40 \mathrm{~s}<\mathrm{T}<150 \mathrm{~s})$. The filtered traces from each station were then stack plotted on the computer screen with resolution such that individual cycles of the Pi 2 waveform could be resolved. At least three stations in the network must have recorded the event to be considered. The earliest time of all the stations observing the event was taken as the Pi 2 onset time and written to a digital file. In the final step the list of Pi 2 onsets was associated with the list of sharp AL onsets. Any AL onset without a Pi 2 onset within plus or minus 20 min was dropped. Similarly, any Pi 2 onset without a corresponding negative bay in AL was dropped. In the case of multiple Pi 2 onsets near a single AL onset, the closest Pi 2 onset was taken as the main substorm onset.

[13] This procedure has been shown to organize the variations in tail magnetic field $H s u$ and McPherron [1996, 1998]. A substorm onset-timing example using the IGS chain is presented in Figure 4. In this figure the vertical line indicates the substorm onset time determined by our procedure.

\subsection{Database for Spring of $\mathbf{1 9 7 8}$ and $\mathbf{1 9 7 9}$}

[14] This spring database covers the period from March to April in 1978 and 1979 when the ISEE spacecraft passed through the tail. Three different sets of observations are used in this database: ground observations for substorm timing, solar wind observations for possible triggers, and magnetotail observations for classification of the type of magnetic activity. One of the advantages of this database is that the ISEE observations in the tail can help assure us that events classified as "not triggered" are actually substorms. Unfortunately, for this dataset the solar wind monitor IMP-8 was seldom near the Earth-Sun line ( $\rho \geq 10 \mathrm{Re}$ ), as required to test the Lyons et al. [1997] hypothesis. Therefore to examine the region closer to the Earth-Sun line, we need another database. For this purpose we will use the ISEE-2 IMF observations in the fall season of 1978 and 1979 when the spacecraft was just upstream of the bow shock. 


\section{Substorm Onset Timimg Event March-30-1978 21h40m}
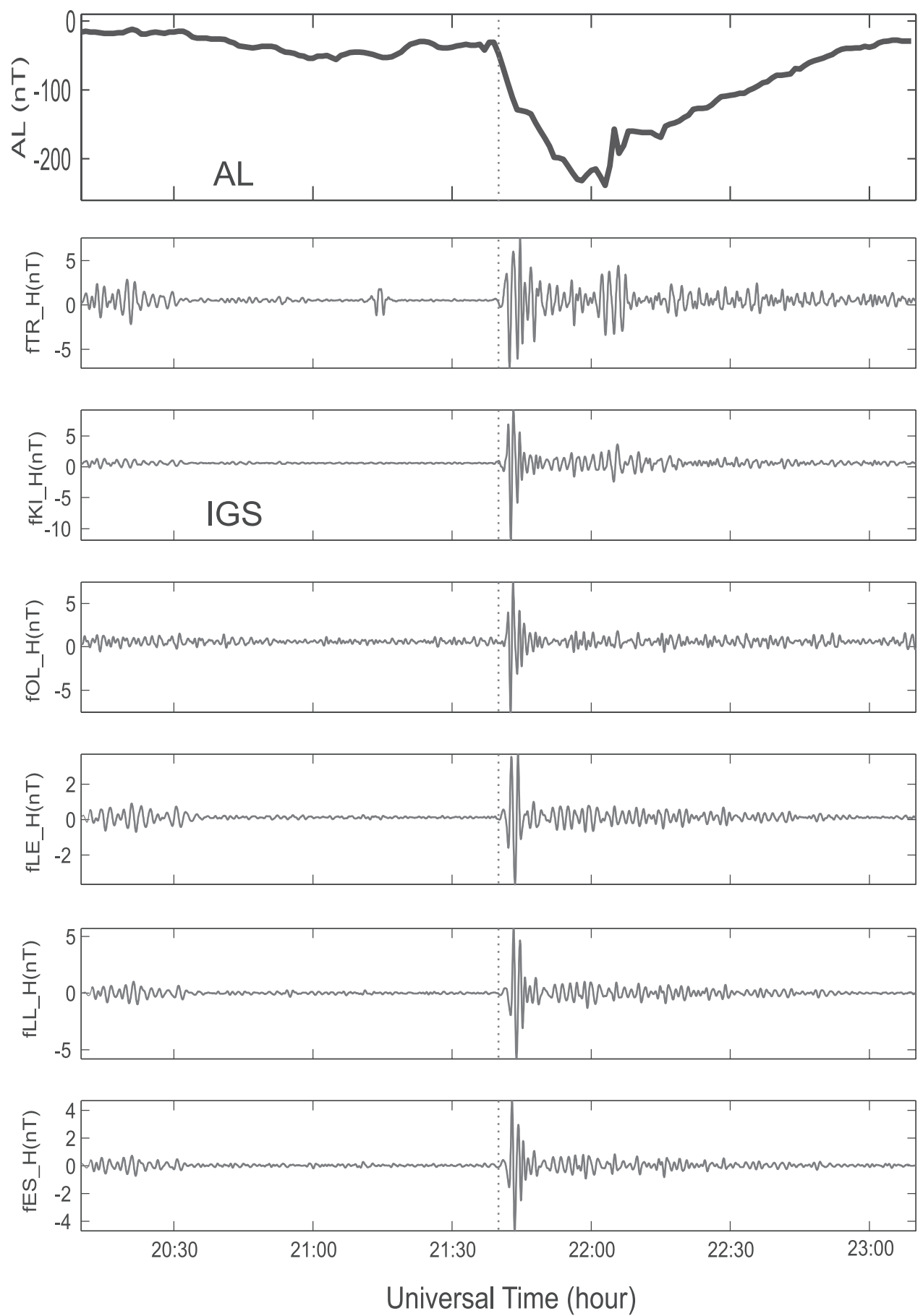

Figure 4. A substorm onset timing example. These IGS station observations are organized from the highest latitude to the lowest latitude [see Stuart, 1982]. There is only one clear Pi 2 burst through out the chain which occurs at 2140 UT. A second Pi 2 can be seen 2200 UT but cannot be seen in the other station and therefore is dropped. The vertical line indicates the onset of the first Pi 2 pulsation.

\subsubsection{Ground Observations (AL and Pi 2s)}

[15] The purpose of the ground observations is to time the substorm onsets. The timing procedure has been described in detail in Figure 4. AE indices are used to determine the time of a sudden intensification of the westward electrojet. AFGL and IGS magnetometer network data are then used to find the start of Pi 2 pulsation bursts, which can more precisely time the substorm onsets. Altogether, $951 \mathrm{AL}$ onsets were identified for which Pi 2 timing was possible for 462 events.

\subsubsection{Solar Wind Observations (IMP-8)}

[16] The orbit of IMP-8 is presented in Figure 5. The IMP-8 satellite has a nearly circular orbit with a radius of about $35 \mathrm{R}_{\mathrm{e}}$. However, the IMP-8 orbit is inclined with 


\section{IMP8 Orbit}
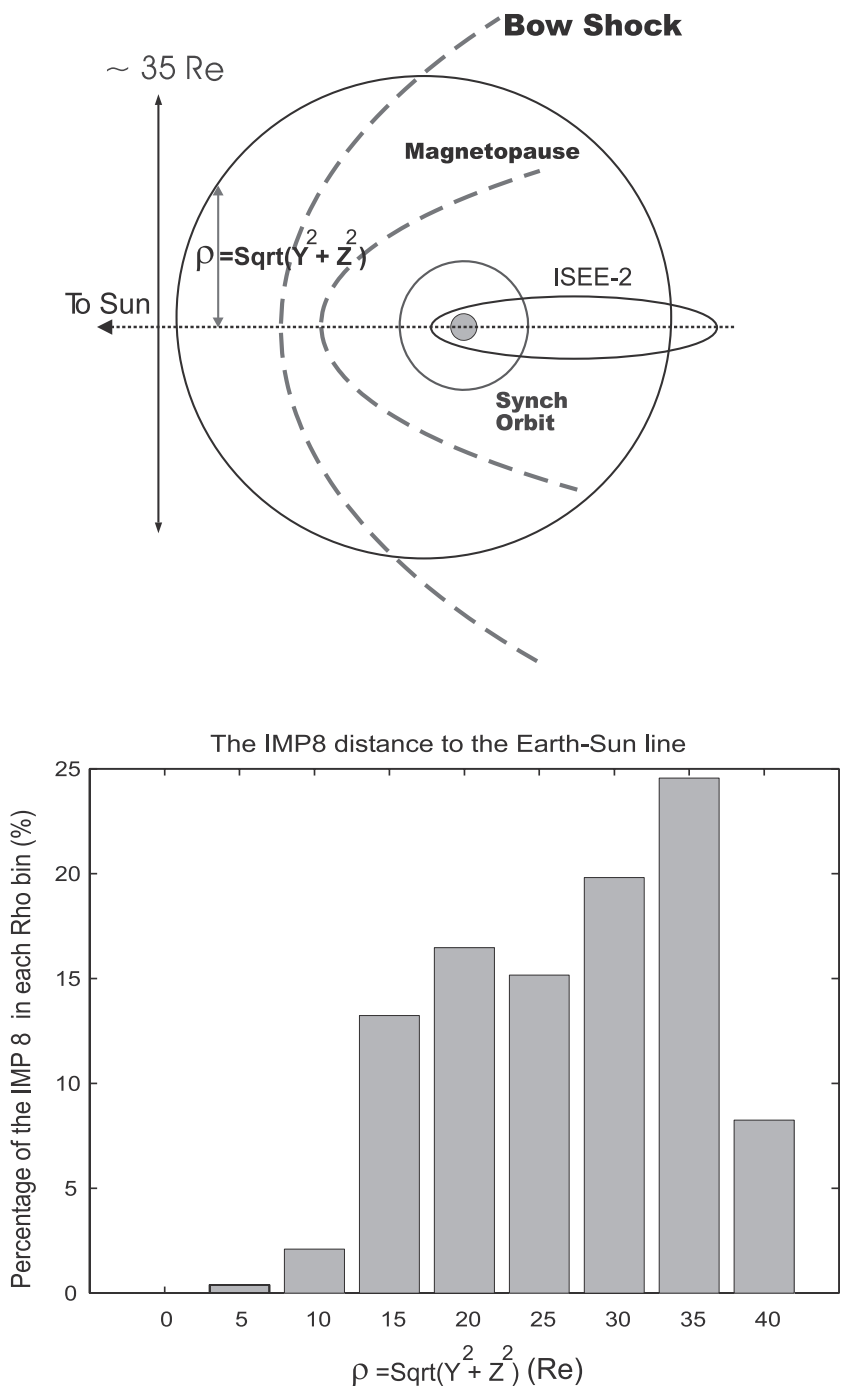

Figure 5. A schematic of the IMF 8 orbit in spring 1978 and 1979. IMP-8 has a nearly circular orbit with radius about $35 \mathrm{R}_{\mathrm{e}}$. At this time ISEE-2 was in the tail with apogee at $22 \mathrm{Re}$. Bottom panel shows the distribution of $\rho$ value for IMP-8 solar wind observation during springs of 1978 and 1979. It is clear that IMP-8 rarely came closer that $\sim 12 \mathrm{R}_{\mathrm{e}}$ to the Earth-Sun line at this time of year.

respect to the ecliptic plane so that in spring it is above the ecliptic when it crosses the Earth-Sun line. Because of this the satellite seldom observed the region with radius $5 \sim 10$ $\mathrm{R}_{\mathrm{e}}$ from the Earth-Sun line. To demonstrate this we have calculated the parameter $\rho$ defined as IMP-8 $\rho=\sqrt{y^{2}+z^{2}}$. The projection of this distance on the ecliptic plane is shown in Figure 5 by an arrowed line orthogonal to the Earth-Sun line. The distribution of values of $\rho$ obtained for IMP-8 solar wind data during springs of 1978 and 1979 is shown in the bottom of Figure 5.

\subsection{Database for Fall of 1978 and 1979}

[17] While IMP-8 has a near circular orbit around the Earth, ISEE-2 has a very eccentric orbit. The schematic orbit and distribution of $\rho$ can be seen in Figure 6. As illustrated, the perigee of ISEE-2 shifts to the dayside during the fall season, providing an opportunity to examine the small-scale hypothesis of Lyons et al. [1997].

[18] In this study we established a fall database that covered the period of October in 1978 and September to October in 1978 and 1979. Two different data types are used: ground observations for substorm timing and solar wind observations for possible triggering. For this dataset the solar wind magnetic field is provided by ISEE-2.

\subsubsection{Ground Observations (AL and $\mathrm{Pi} 2 \mathrm{~s}$ )}

[19] The substorm timing procedure is the same as used for the spring database. However, for this study we only

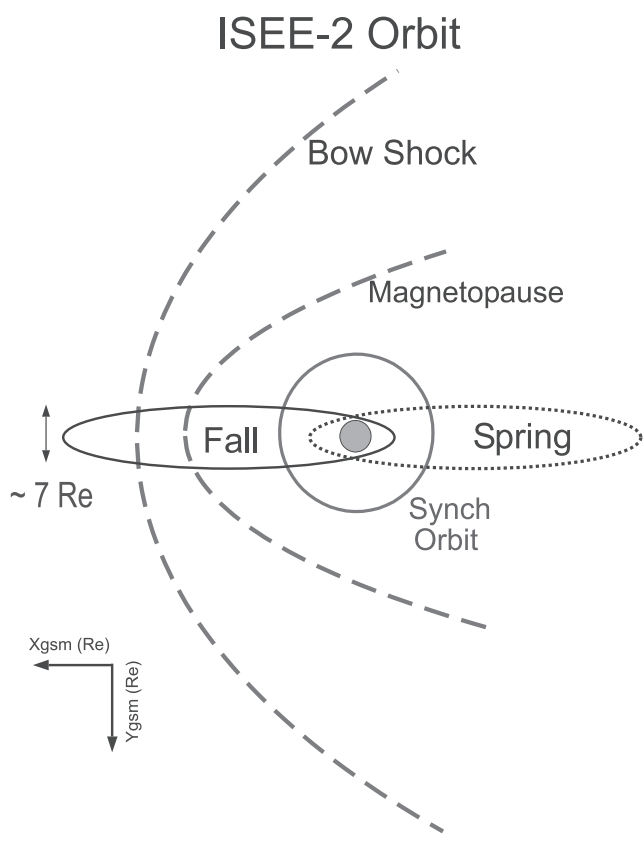

The ISEE-2 Distance to The Earth-Sun Line

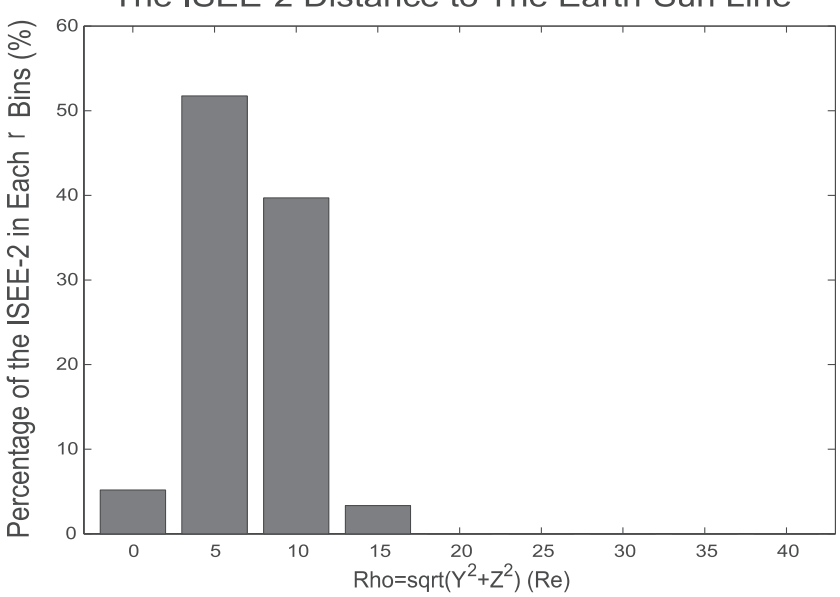

Figure 6. A schematic of the ISEE-2 orbit in fall 1978 and 1979. The ISEE-2 orbit has a semi minor axis only about $7 \mathrm{R}_{\mathrm{e}}$ long and samples the solar wind upstream of the subsolar point on the bow shock. At the bottom of this figure a histogram of the perpendicular distance of ISEE-2 from the Earth-Sun line during the interval used in the study. It is clear that ISEE-2 was within $\sim 10 \mathrm{R}_{\mathrm{e}}$ of the Earth-Sun line most of the time. 
used the AFGL magnetometer network data because its observations are more reliable than the IGS observations. Altogether we identified 599 AL onsets for which we were able to more precisely time 251 using AFGL Pi 2 data.

\subsubsection{Solar Wind Observations (ISEE-2)}

[20] Because of its highly elliptical orbit, ISEE-2 was just in front of the subsolar bow shock during the fall period of its operation (Figure 6). In contrast to the situation for IMP-8, ISEE-2 was close to the Earth-Sun line in fall. From the distribution of $\rho$ in Figure 6 it is clear that ISEE-2 IMF observations fill the gap present in the IMP-8 solar wind observations during spring.

\subsection{Propagation of Solar Wind Observations}

[21] For the present study the ideal position to observe solar wind conditions is at a point just upstream of the merging region where energy enters the magnetosphere from the solar wind. Unfortunately, this situation never occurs with IMP-8. Consequently, we have to modify the solar wind time series to account for the change in time delay between corresponding events occurring at IMP-8 (or ISEE-2) and the coupling region. To do this, we have estimated the actual time delay using a formula developed by Zwickl and coworkers [cf., Baker et al., 1983]. Here, the formula has been expressed ignoring the orbital motion of the earth and using a small-angle approximation:

$$
T_{\text {solar wind }}=\frac{\left(X-X_{0}\right)}{V_{s w}}+\frac{\left(Y-Y_{0}\right) \cdot T_{0}}{2 \pi a}
$$

where $T_{\text {solar wind }}$ is the solar wind time delay, $X$ and $Y$ are the location of the spacecraft in GSM coordinates, $X_{0}$ and $Y_{0}$ are the coordinates of the subsolar point on the bow shock, $T_{0}$ is the mean solar rotation period, $a$ is the mean radial distance from the Sun to the earth, and $\mathrm{V}_{\mathrm{sw}}$ is the instantaneous solar wind bulk speed. Using the average position of the subsolar bow shock $\left(X_{0}=15, Y_{0}=0\right)$ as a fixed reference point we can approximately eliminate effects of solar wind propagation. In this approximation we have assumed that discontinuities in solar wind properties are aligned along the average spiral angle of the solar wind and are not propagating.

[22] The solar wind is slowed by the bow shock as it passes into the magnetosheath. Our estimate of the time delay in the magnetosheath is $4 \mathrm{~min}$. Once the solar wind arrives at the magnetopause, we assume another $2 \mathrm{~min}$ as the propagation time to the Earth. Thus the solar wind is time propagated to the Earth by a formula $T_{\text {prop }}=T_{\text {solar wind }}+T_{\text {magnetosheath }}+$ $T_{\text {field line, where }} T_{\text {solar wind }}$ is the solar wind propagation time from satellite to Earth, $T_{\text {magnetoshearth }}(\sim 4 \mathrm{~min})$ is the propagation time from bow shock to magnetopause, and $T_{\text {field_line }}(\sim 2 \mathrm{~min})$ is the propagation time from magnetopause to Earth. The procedure for calculating the solar wind propagation time delay is shown schematically in Figure 7.

[23] In this paper we have tried our best to estimate the time propagation error from the solar wind monitor (ISEE-2) to the Earth-Sun line. Unfortunately, the ISEE-2 plasma instruments failed in June 1978 so it was not possible to get the solar wind velocity from the ISEE-2 plasma instrument. Plasma data are available from the IMP 8 spacecraft, but only rarely were both spacecraft in the solar wind at the same time. An examination of the measured IMP-8 values of speed during the study interval gave 295, 390, and
$485 \mathrm{~km} / \mathrm{s}$ for the median and quartiles of speed. Thus we used a solar wind speed of $400 \mathrm{~km} / \mathrm{s}$ to propagate the solar wind to the magnetopause in the ISEE data. The difference in velocity between the upper and lower quartiles and the median causes about $30 \mathrm{~s}$ difference in time delays. Since the time resolution of the data is one minute the errors caused by using the median solar wind speed to time propagate the data are negligible.

[24] It should be noticed that this study is using only one solar wind monitor. It has been suggested that two or more solar wind monitor should be used to estimate a more accurate arrival time of the IMF structure [e.g., Blanchard et al., 2000]. Unfortunately, there is no extra solar wind monitor near the earth available for the arrival time adjustment. Yet the fact that the overall statistics for the IMP 8 (little away from the Earth-Sun line) and the ISEE-2 (almost parallel to the Earth-Sun line) is almost the same may suggest that we did not miss many solar wind perturbations statistically.

\subsection{Visual Identification of Triggered and Nontriggered Substorms}

2.5.1. Procedure for Visually Classifying Substorms as Either Triggered or Nontriggered

[25] The question we want to answer is how often a northward turning of the IMF triggers a substorm. As a first approximation to this probability, we developed a simple procedure for classifying IMF characteristics at substorm onset. The steps in this procedure included the following:

[26] Determining substorm onset time. Substorm onset times were determined by AL index and Pi 2 pulsations as described in Figure 4.

[27] Plotting IMF components. The IMF components $B_{z}$ and $\mathrm{B}_{\mathrm{y}}$ and the solar wind dynamic pressure $\mathrm{P}_{\mathrm{dyn}}$ were plotted at $\sim 1$-min resolution. The solar wind propagation delay at the time of onset was subtracted from the substorm onset time and plotted on the graph as a vertical line.

[28] Evaluating sufficiency of data points. A time window of $\pm 45 \mathrm{~min}$ around onset time was used to check whether enough data points were available to examine the triggering process. At least $70 \%$ of the data in this window must be good records.

[29] Scanning the data for northward turnings of $B_{z}$ defined as events that satisfy the following criteria: (1) If at least a $2 \mathrm{nT}$ northward turning of IMF $\mathrm{B}_{\mathrm{z}}$ is associated with an onset within $\pm 10 \mathrm{~min}$ and $\mathrm{B}_{\mathrm{z}}$ does not fluctuate more than $\pm 2 \mathrm{nT}$ within \pm 20 min of onset, then the onset will be classified as a " $\mathrm{B}_{\mathrm{z}}$ triggered substorm." (2) If no $\mathrm{B}_{\mathrm{z}}$ perturbations are present but there is at least a $2 \mathrm{nT}$ reduction of IMF $\left|B_{y}\right|$ associated with an onset within $\pm 10 \mathrm{~min}$, and $\mathrm{B}_{\mathrm{y}}$ does not fluctuate more than $\pm 2 \mathrm{nT}$ within $\pm 20 \mathrm{~min}$ of onset, then the interval will be called a " $\mathrm{B}_{\mathrm{y}}$ triggered event." (3) If the solar wind plasma pressure increases as a step function and its change in magnitude is more than $7 \mathrm{nP}$, the event will be called a "pressure triggered" event. It should be noticed that this criterion is an attempt to find whether large solar wind "pressure" changes can cause substorm onset. The magnitude of this change was selected to be several times the median dynamic pressure of the solar wind, $2.4 \mathrm{nP}$. (4) If none of the above perturbations can be found, then the event will be designated a "nontriggered event." 


\section{Proceduare to calculate the propagation delay}

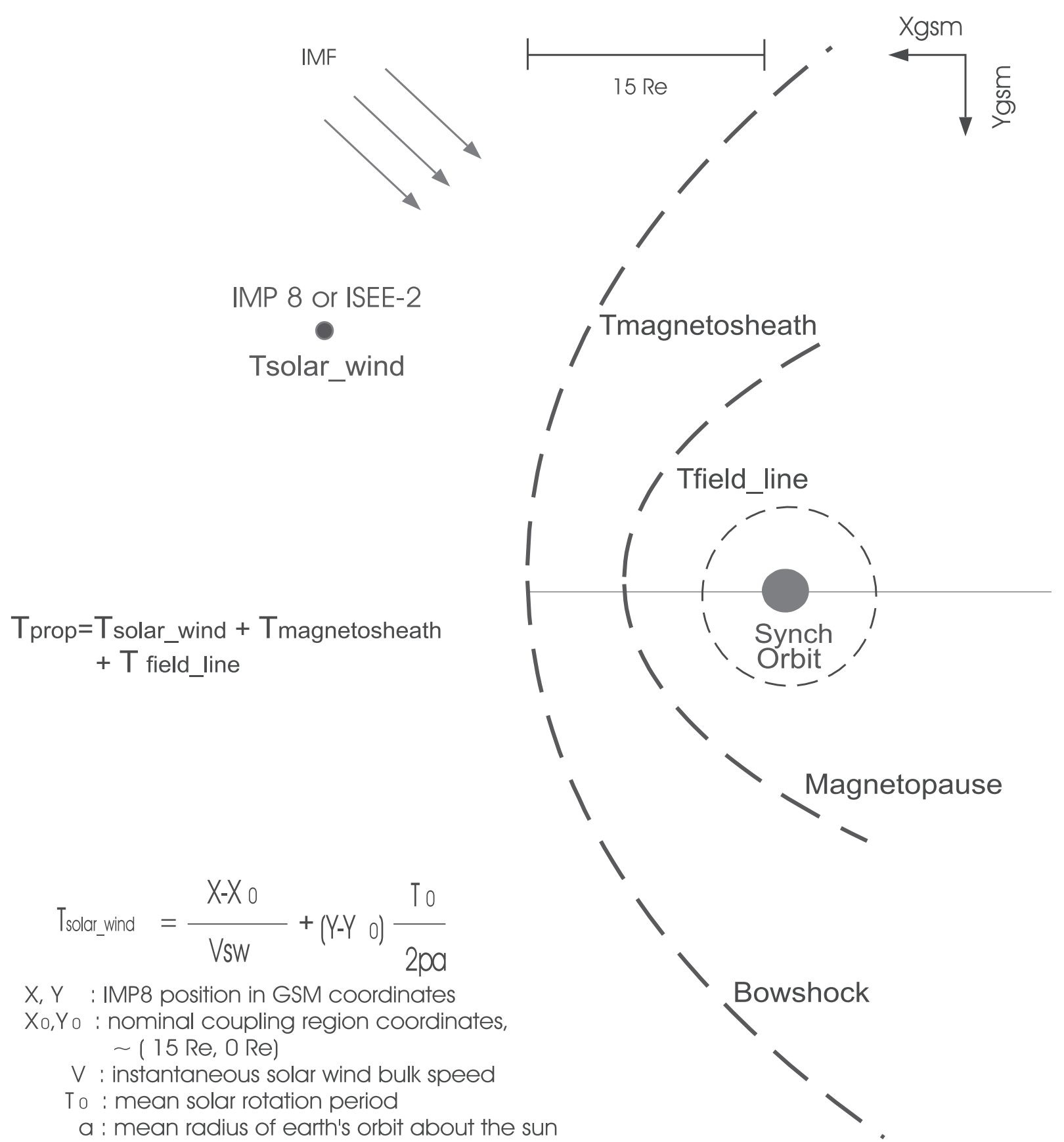

Figure 7. A summary of the method used for the calculation of solar wind time delay between the solar wind monitor and the surface of the Earth. $\mathrm{T}_{\text {magnetosheath }}$ is the propagation delay from bow shock to magnetopause $(\sim 4 \mathrm{~min}) . \mathrm{T}_{\text {field_line }}$ is the propagation delay from magnetopause to the Earth $(\sim 2 \mathrm{~min})$.

\subsubsection{IMP-8 Examples Showing a Triggered and Nontriggered Substorm}

[30] Figure 8 shows examples of triggered and nontriggered substorm onsets selected according to the above procedure. The left panel presents an event on 7 March 1978. In this example, after time propagating the solar wind observations, we can see a clear northward turning of IMF
$\mathrm{Bz}$ very close to the propagated onset time. Note there was also a decrease of $\left|B_{y}\right|$, but we classify this as a $B_{z}$ triggered event. The solar wind dynamic pressure shows very little change although there were small fluctuations some minutes before and after the propagated onset time. In the right panel, another example on 6 March 1979 is shown. The scale is the same as for the triggered example. It is apparent 
that neither $\mathrm{B}_{\mathrm{z}}$ or $\mathrm{B}_{\mathrm{y}}$ shows any clear perturbations. Also the solar wind dynamic pressure was steady around the substorm onset time. Thus this event was classified as a nontriggered event.

\subsubsection{ISEE-2 Examples Showing a Triggered and Nontriggered Substorm}

[31] During the fall period of 1978 and 1979 no plasma data were available on the ISEE spacecraft. Thus we are unable to examine the occurrence frequency of the "pressure trigger" events. However, it is believed that this "pressure trigger" class does not occur frequently [e.g., McPherron et al., 1986]. Given this result, we only considered the IMF $\mathrm{B}_{\mathrm{z}}$ and $\mathrm{B}_{\mathrm{y}}$ triggers in the ISEE-2 observations.

[32] In Figure 9 the left panel shows an event classified as an IMF $B_{z}$ triggered substorm. A northward turning of $B_{z}$ appears to be associated with substorm onset at $1352 \mathrm{UT}$. The IMF $\left|B_{y}\right|$ also shows a reduction $\sim 4$ min ahead of substorm onset. According to our procedure, this was classified as $\mathrm{a}_{\mathrm{z}}$ triggered event. The right panel of Figure 9 shows a nontriggered event. Neither IMF $B_{z}$ or $\mathrm{B}_{\mathrm{y}}$ exhibit any clear perturbations at the time of the onset.

[33] The ground observations for these triggered and none triggered examples are shown in Figure 10. The substorm onset timing by using AL index and Pi 2 pulsation has been added in this figure for comparison. It is clear that all of the examples have a sudden break of AL index and its associated Pi 2 enhancement. However, the IMF $B_{z}$ around the substorm onset seems very different.

\subsection{Occurrence Frequency of Triggered and Nontriggered Substorms}

\subsubsection{IMP-8 Observations}

[34] Out of almost five hundred Pi 2 timed substorm onsets we find there were only about two hundred events with IMF available near the onset times. The occurrence frequencies for various classes of possible triggers are presented in Table 1 below.

[35] A graphical summary of these results is presented in Figure 11. It is clear in these statistics that IMF triggered substorms (including $\mathrm{B}_{\mathrm{z}}$ or $\left|\mathrm{B}_{\mathrm{y}}\right|$ ) occur frequently. However, we also find that for about $40 \%$ of the substorms we cannot identify an apparent trigger around the time of substorm onset. Some of the identified events even occurred during situations in which the IMF was northward for at least $\pm 20 \mathrm{~min}$ before and after substorm onset. It is very hard to imagine these substorm events can have been initiated by a mechanism that reduces the westward electric field across the magnetosphere. In summary, even with more precise substorm onset timing, we find that our statistical results are consistent with those in the study of McPherron et al. [1986], which relied heavily on the auroral indices. Apparently, the inclusion of IMF $\left|B_{y}\right|$ does not significantly change the statistical results.

\subsubsection{Dependence of Triggering on Location of Solar} Wind Monitor

[36] As mentioned above, another argument raised by Lyons et al. [1997] is that the solar wind monitor must be in the correct position to detect IMF triggers. The authors suggested that the probability of identifying IMF triggers decreases as the distance of the monitor from the Earth-Sun line increases. To determine whether this is the case we have calculated the probability of classifying a substorm onset as either triggered or nontriggered as a function of the distance of IMP-8 from the Earth-Sun line.

[37] Figure 12 summarizes the IMP- 8 results as a function of distance from the Earth-Sun line. The upper left panel shows the probability that IMP-8 was in a particular $5 \mathrm{Re}$ wide bin of $\rho$. Since this $\rho$ distribution is calculated for all IMP8 solar wind observations, the percentage in each $\rho$ bin can represent the time IMP 8 spent in that particular $\rho$ bin. These distributions will be used as our approximation for the time distribution for IMP 8 solar wind observation in every $\rho$ bin during our selected substorm events. The peak at $35 \mathrm{Re}$, corresponding to the radius of the IMP-8 orbit, is a consequence of the definition of $\rho$. The IMP- 8 spacecraft in circular orbit spends the longest time in the bin at the ends of a diameter passing through the center of the orbit. Thus we expect that most of the events of either type will be observed in this bin. The upper right panel of Figure 12 shows the total number of observed events of each type as a function of $\rho$. As expected, the maximum number of occurrences of either class of events occurs in the $35 \mathrm{Re}$ bin. Because of this we must weight the number of events observed in each bin by the reciprocal of the fraction of time it spends in a given bin. This "time normalized ratio" for both classes of events is defined as follows:

$$
f_{\rho}=\frac{n_{\rho}}{N_{t o t}}
$$

$$
f_{T_{\rho}}=\frac{t_{\rho}}{T_{t o t}}
$$

$$
R_{\rho T}=\frac{f_{\rho}}{f_{T}}=\frac{n_{\rho}}{N_{t o t}} \cdot \frac{T_{t o t}}{t_{\rho}},
$$

where $\rho$ is the distance from IMP- 8 to Earth-Sun line, $n_{\rho}$ is the number of triggered or nontriggered substorms for each $\rho$ bin, $N_{\text {tot }}$ is the total number of triggered or nontriggered substorms in this analysis, $t_{\rho}$ is the time IMP- 8 spent in each $\rho$ bin, and $T_{\text {tot }}$ is the total time of solar wind observation in this analysis, $f_{\rho}$ is the occurrence probability for triggered or nontriggered substorms in each $\rho$ bin, $f_{T_{\rho}}$ is the fraction of time IMP-8 spent in each $\rho$ bin. $R_{\rho T}$ is the time-normalized ratio for each $\rho$ bin for triggered or nontriggered events. It should be noticed that the time-normalized ratio is not a probability function. This ratio is shown in the lower left panel of Figure 12. The most obvious feature in this plot is that, except for fluctuations probably due to noise, the ratio for both triggered and nontriggered substorms is near 1.0. From the definition of this ratio in equation (4) it can be seen that this implies that the number of events of a given type observed in a bin is proportional to the time the spacecraft spends in the bin. Furthermore, the constant of proportionality does not change with $\rho$, contrary to the Lyons hypothesis. Figure 12 In the lower right panel of the figure we plot the probability of observing triggered and nontriggered substorms in each $\rho$ bin. In this panel, probability is defined as the ratio of the number of events of a given type to the total number of events of both types observed in a particular bin. Apart from the two extreme bins that have very few observations, the probabilities of observing triggered and nontriggered events change very 
IMF Triggered Event 03/07/78 22h05m

$\mathrm{X}: 20.48$ Y:10.53 Z: -15.3 rho:18.57 (Re)

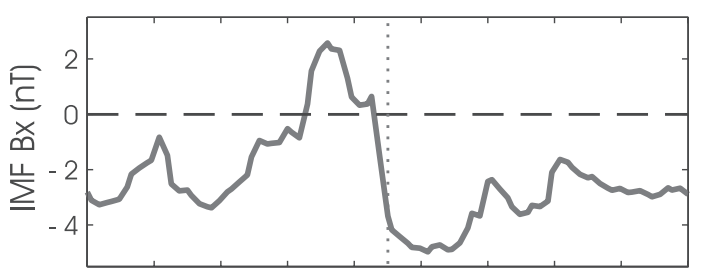

timed by IGS Pi 2
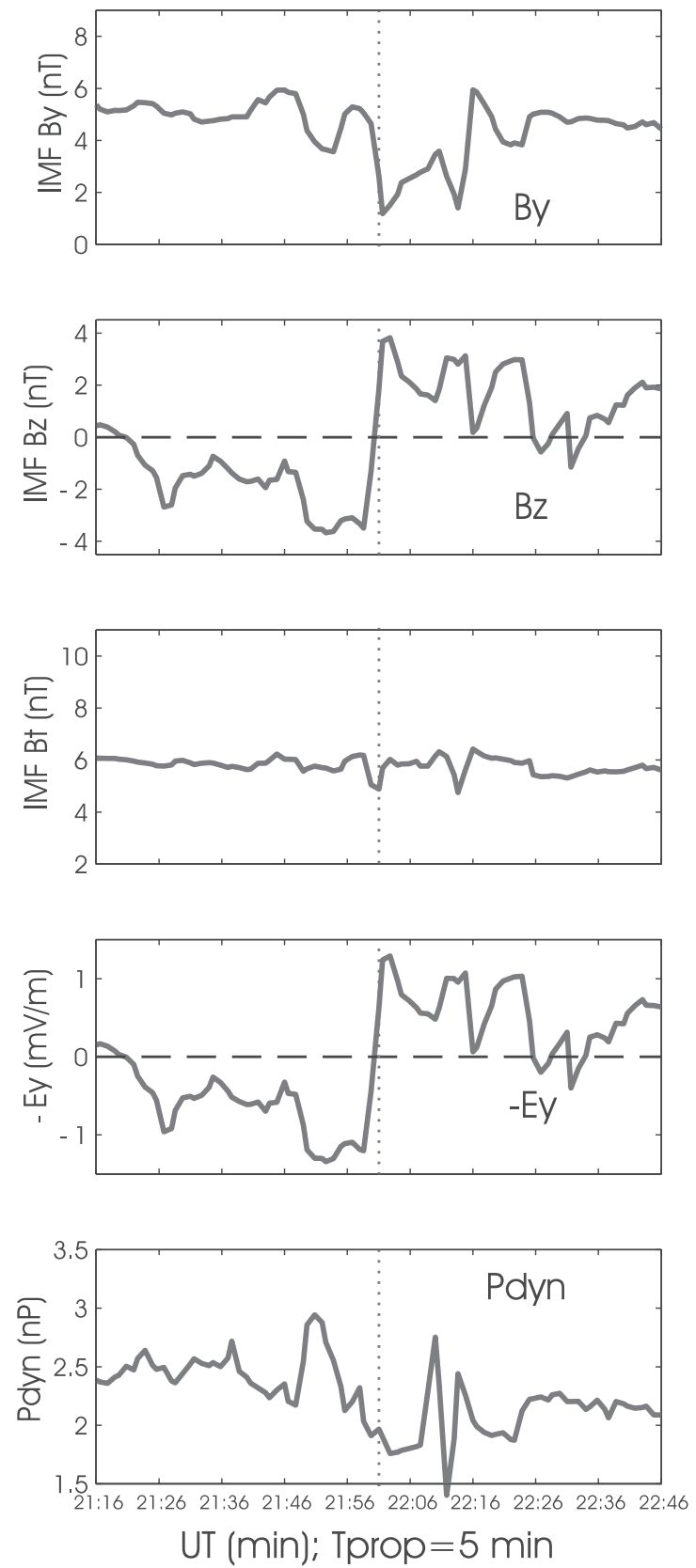

IMF Non-Triggered Event 03/06/79 20h42m X:19.68 Y:-9.75 Z:-22.14 rho:24.19 (Re)

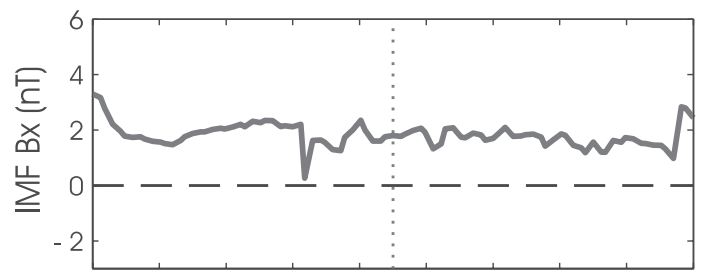

timed by IGS Pi 2
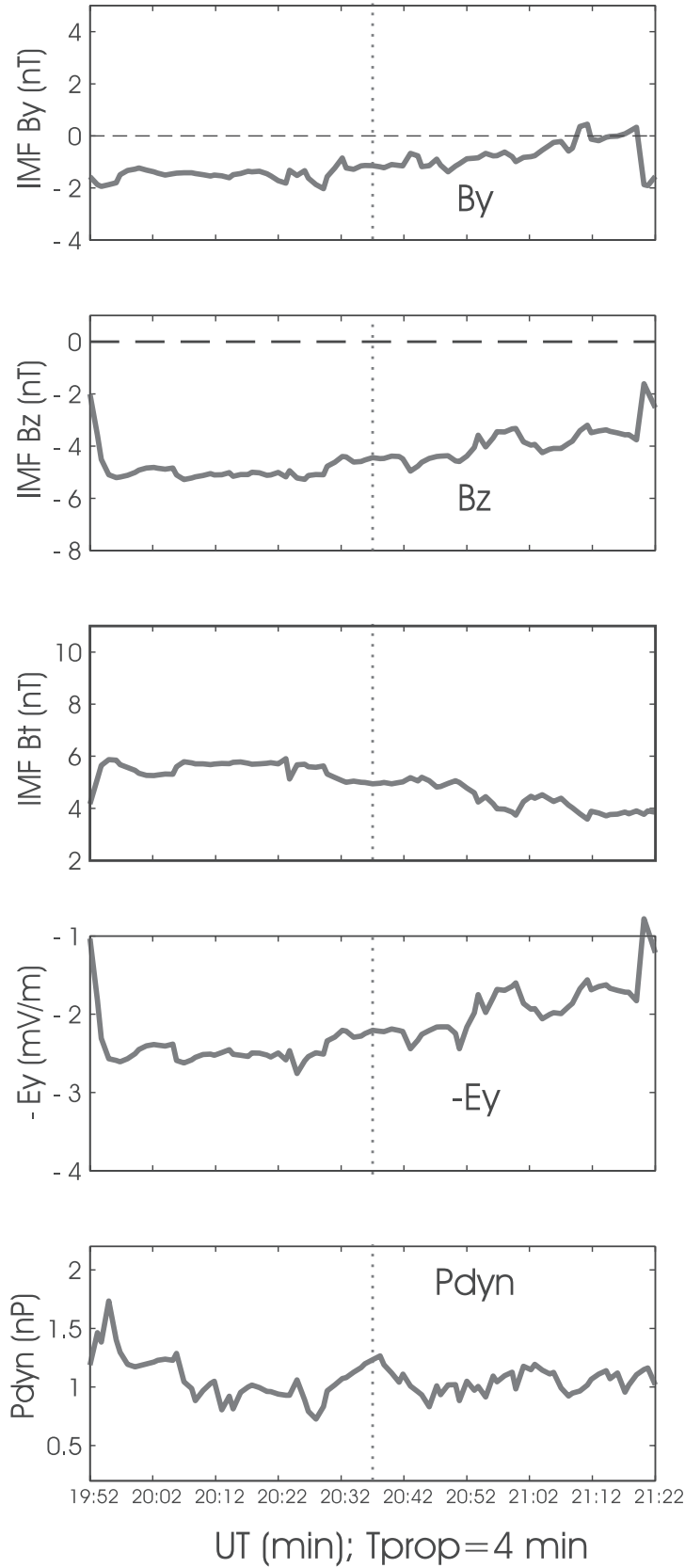

Figure 8. Examples of the IMF data for triggered and nontriggered substorm events selected in the IMP-8 study. The left panel shows a triggered event. The IMF $B_{z}$ is apparently associated with a substorm onset at 2205 UT on 7 March 1978. The right panel shows an example of a nontriggered event. There is no apparent change in any IMF component in \pm 40 min around substorm onset. 
ISEE-2 IMF Trigger Example (by AFGL Pi 2) 10/21/1978 UT 13h52m
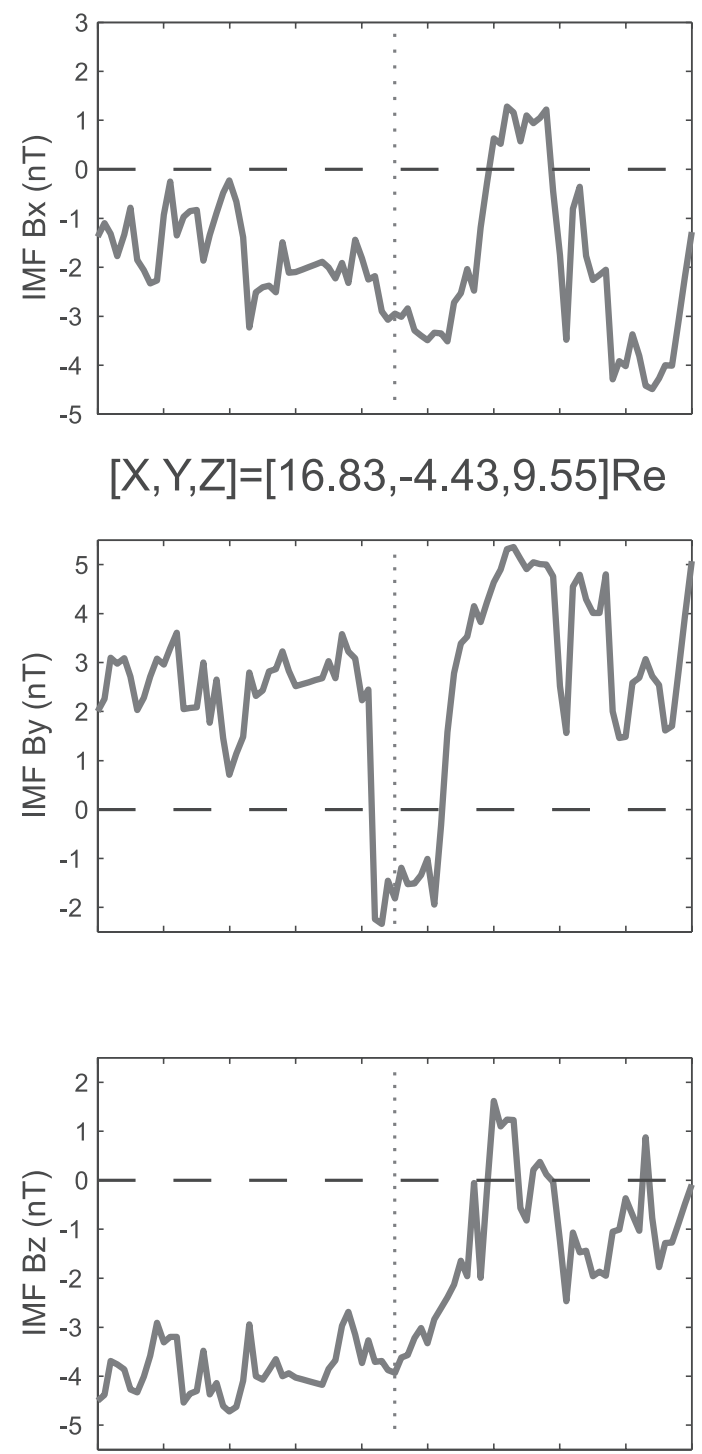

Tprop $=5 \mathrm{~min}$

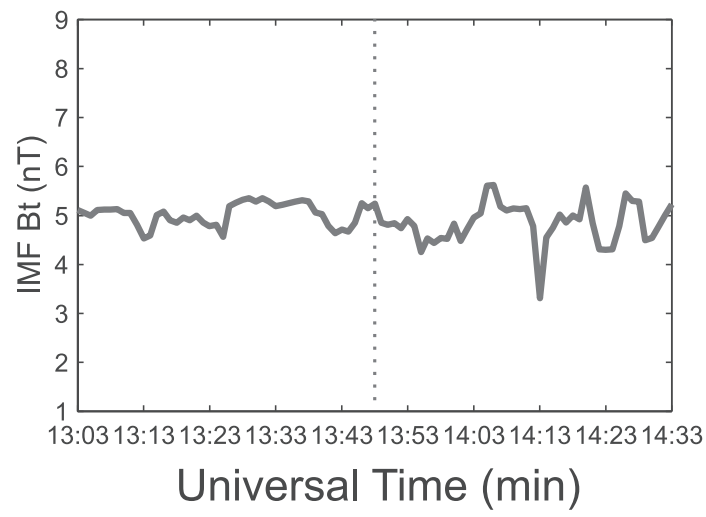

ISEE-2 IMF Non-Trigger Example (by AFGL Pi 2) 10/15/1979 UT 12h50m
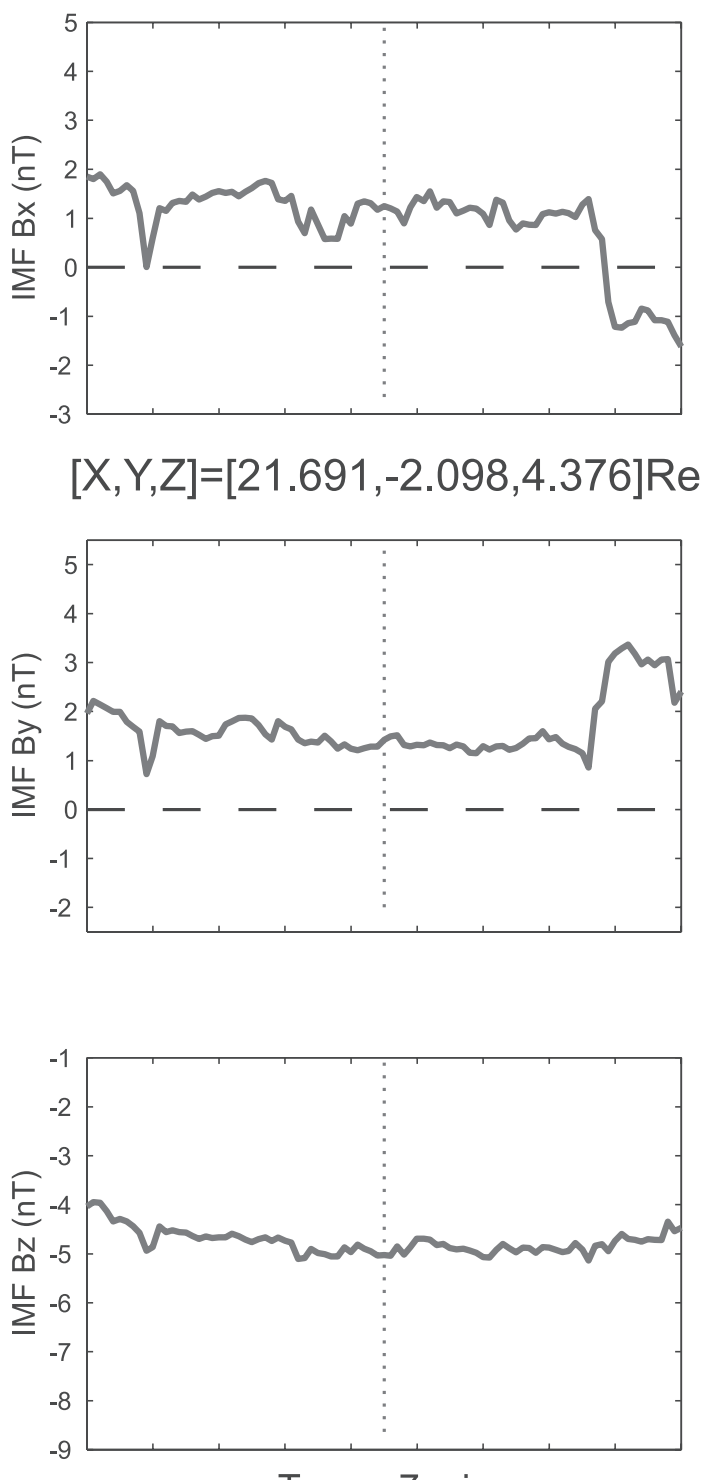

Tprop=7 $\mathrm{min}$

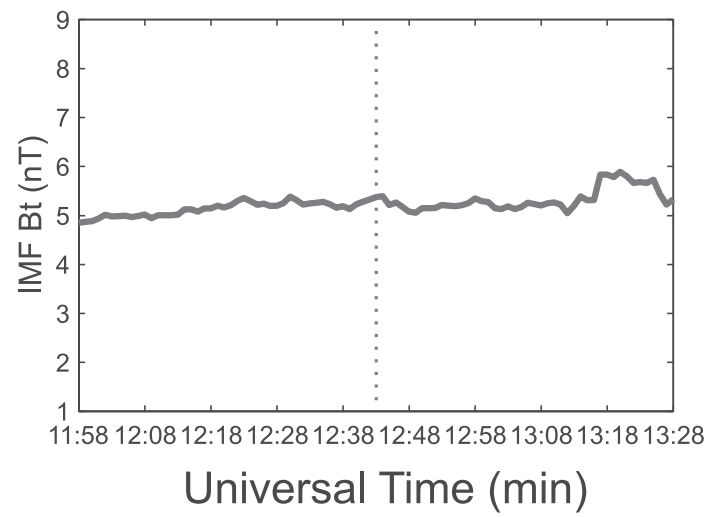

Figure 9. Examples of triggered and nontriggered events selected in the ISEE-2 study. The left panel shows an example of an event that has an apparent IMF $B_{z}$ trigger at substorm onset. The right panel shows an example of a nontriggered event for which there is no change in any component of the IMF for sometime before and after the onset. 
Table 1. Fraction of the Substorm Onsets Apparently Triggered by a Particular Type of Change in IMF $\mathrm{B}_{\mathrm{z}}$ or $\mathrm{B}_{\mathrm{y}}$ Observed by the IMP-8 Spacecraft

\begin{tabular}{lr}
\hline Classification of Possible Trigger & Number, $\%$ \\
\hline Total substorm events with IMF data & $215,100 \%$ \\
Events possibly triggered by IMF $\mathrm{B}_{\mathrm{Z}}$ & $100,47 \%$ \\
Events possibly triggered by IMF $\left|\mathrm{B}_{\mathrm{y}}\right|$ & $23,10 \%$ \\
Dynamic pressure triggered events & $7,3 \%$ \\
Nontriggered events (IMF $\left.\mathrm{B}_{\mathrm{z}}<0\right)$ & $60,29 \%$ \\
Nontriggered events (IMF $\mathrm{B}_{\mathrm{z}}>0, \pm 20$ minutes around onsets) & $25,11 \%$ \\
\hline
\end{tabular}

little with $\rho$. However, if the apparent trends are significant, then they indicate a higher probability of observing triggered events at the largest distance from the Earth-Sun line.

[38] The original small-scale structure hypothesis of Lyons et al. [1997] suggests that the probability of observing IMF triggers increases as the monitor approaches the Earth-Sun line. Our results, however, indicate that it is constant or decreases over the range of observations possible for IMP-8. However, as pointed out by L. R. Lyons (personal communication, year?), it is still possible that there is a rapid increase in the probability of detecting triggers in the range $\rho<=15 \mathrm{Re}$. To examine this hypothesis further, we use another statistical study to investigate the region with $\rho$ close to the Earth-Sun line.

\subsubsection{ISEE-2 Observations}

[39] As shown in the previous section, we need observations closer to the Earth-Sun line to confirm or deny the small-scale structure hypothesis. ISEE-2 IMF observations provide a good opportunity to do this in the fall seasons of 1978 and 1979. During these intervals the ISEE spacecraft has its apogee near the Earth-Sun line. The median $\rho$ for these intervals is only about $7 \mathrm{R}_{\mathrm{e}}$ (see Figure 6), which is exactly the region missing from the IMP- 8 observations.

[40] Using the same procedure as in the IMP-8 study we identified 145 substorm onsets accurately timed with Pi 2 data and with ISEE nearly upstream of the subsolar bow shock. After associating the ISEE-2 IMF observations with substorm onsets, we found that the probability of observing the different classes of relationship between the IMF and substorms is virtually the same as for the IMP-8 spacecraft. These results are summarized in Figure 13 and Table 2.

\subsubsection{Comparison of the Occurrence Frequency for} Two Satellites

[41] The results from the ISEE database and a comparison with similar results from IMP-8 are presented in Table 2. In this comparison, it is very clear that both satellites observed essentially the same occurrence probabilities for all classes of events. While it appears that the ISEE triggering probability is slightly higher than the IMP-8 probability $(61 \%$ versus $57 \%$ ), the difference is much smaller than expected from the small-scale structure hypothesis. Also, not including pressure triggered substorms in the ISEE study raise these numbers slightly. We therefore conclude that the probability of observing substorm triggering is not a significant function of the distance of the monitor from the Earth-Sun line, at least to distances equal to the apogee of IMP-8 (35 Re).

[42] It should be noted that the foregoing conclusion only dismisses the possibility that small-scale structures in the solar wind must hit the subsolar magnetopause. It is still possible such structures could account for the class of nontriggered events provided their impact somewhere on the dayside magnetopause can trigger a substorm. If this is the case, then the frequency of occurrence of such structures must be very high to account for the $40 \%$ of substorms we classified as nontriggered. If this is the case, it will never be possible to accurately predict geomagnetic activity from upstream observations because a large array of monitors would have to be placed in the solar wind.

\subsection{Automatic Identification of Possible IMF Triggers}

[43] The advantage of the visual procedure used thus far is that it takes advantage of human pattern recognition abilities. It is frequently difficult to write a pattern recognition program that captures the same set of events. The disadvantage is that without automation it is virtually impossible to assess the statistical significance of the inferred association because it is difficult to remove subjective influence from the selection. Also, it would be extremely time-consuming to visually exam every 10 -min interval in a continuous IMF record. Because of this we have developed an automatic detection algorithm using the Lyons et al. [1997] criteria and apply it to the IMP-8 data sets. In this section we use these criteria to identify IMF $B_{z}$ triggers and to examine what effect the selection procedure has on the statistics.

[44] The set of criteria developed by Lyons et al. [1997] is given in the following: (1) the growth phase requirement is $\mathrm{B}_{\mathrm{z}}<0$ for 22 of the previous $30 \mathrm{~min}$, (2) the turning initiation requirement is $\left[\mathrm{B}_{\mathrm{z}}\left(\mathrm{t}_{0}+\Delta \mathrm{t}\right)-\mathrm{B}_{\mathrm{z}}\left(\mathrm{t}_{0}\right)\right] / \Delta \mathrm{t} \geq$ $0.375 \mathrm{nT} / \mathrm{min}$, (3) the sustained northward turning requirement is $\mathrm{S}_{10} \geq 0.175 \mathrm{nT} / \mathrm{min}$, where $\mathrm{S}_{10}$ is the slope of the linear regression to the data between $t_{0}$ and $t_{0}+10 \mathrm{~min}$, where for $0 \leq t_{0}-t \leq 3 \min : B_{z}(t) \geq B_{z}\left(t_{0}\right)+0.15 n T\left[t-t_{0}\right]$ and for $3 \min \leq t_{0}-\bar{t} \leq 10 \min : B_{z}(t) \geq B_{z}\left(t_{0}\right)+0.45 \mathrm{nT}$, and (4) no other point within the previous $10 \mathrm{~min}$ interval satisfies the above criteria.

[45] Figure 14 shows an example result obtained with this automatic selection procedure. The crosses identify the points that satisfy the growth phase requirements, while the dark circles are possible IMF $\mathrm{B}_{\mathrm{z}}$ northward turning triggers. In these criteria a possible northward turning point must be a growth phase point. However, not every growth phase satisfies the other criteria becoming an IMF trigger. In Figure 14 there are three identified IMF triggers. The first two of the IMF triggers occurred during periods satisfying the growth phase criteria. The third IMF trigger occurred at about 0740 UT. The IMF trigger is the first point that satisfies the remaining criteria.

\subsection{Comparison Between Auto and Visual Selected Triggered Events}

\subsubsection{IMP-8 Satellite}

[46] Application of the automatic selection procedure to the IMP-8 data produced a list of 357 possible IMF $B_{z}$ triggers. Apparently, there are more possible IMF $\mathrm{B}_{\mathrm{z}}$ triggers than substorm onsets, since we found only 216 onsets in the same interval. This demonstrates that not all triggers cause substorms. Clearly the magnetosphere must be in the correct state for this to happen.

[47] A comparison of the automatic and visual procedures applied to the same data illustrates the reasons for differ- 
IMF Triggered Event 3/7/78 22h05m

IMF Triggered Event 3/6/79 20h42m
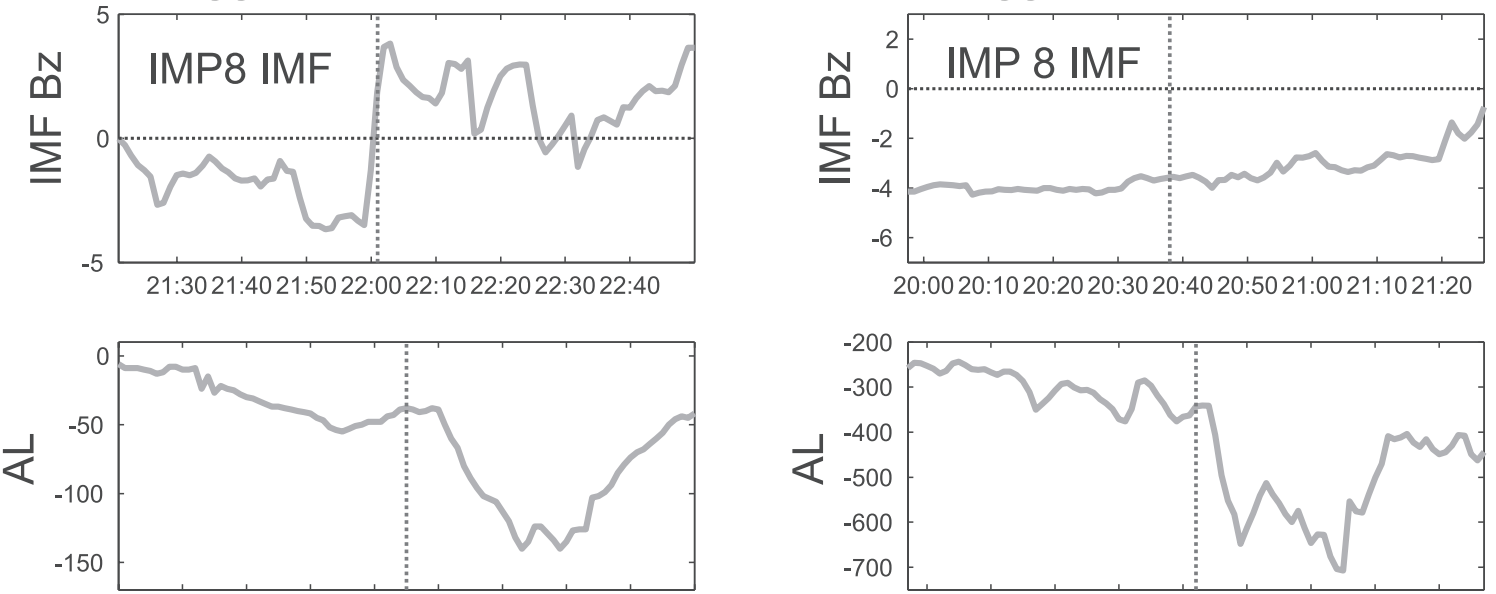

Universal Time

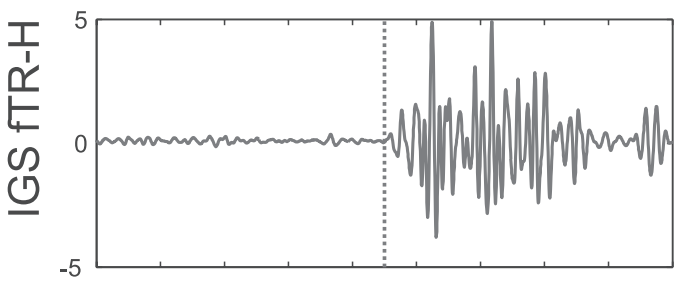

Universal Time

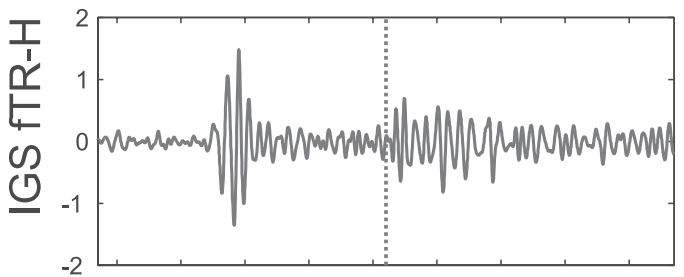

IMF Triggered Event 10/21/78 13h52m
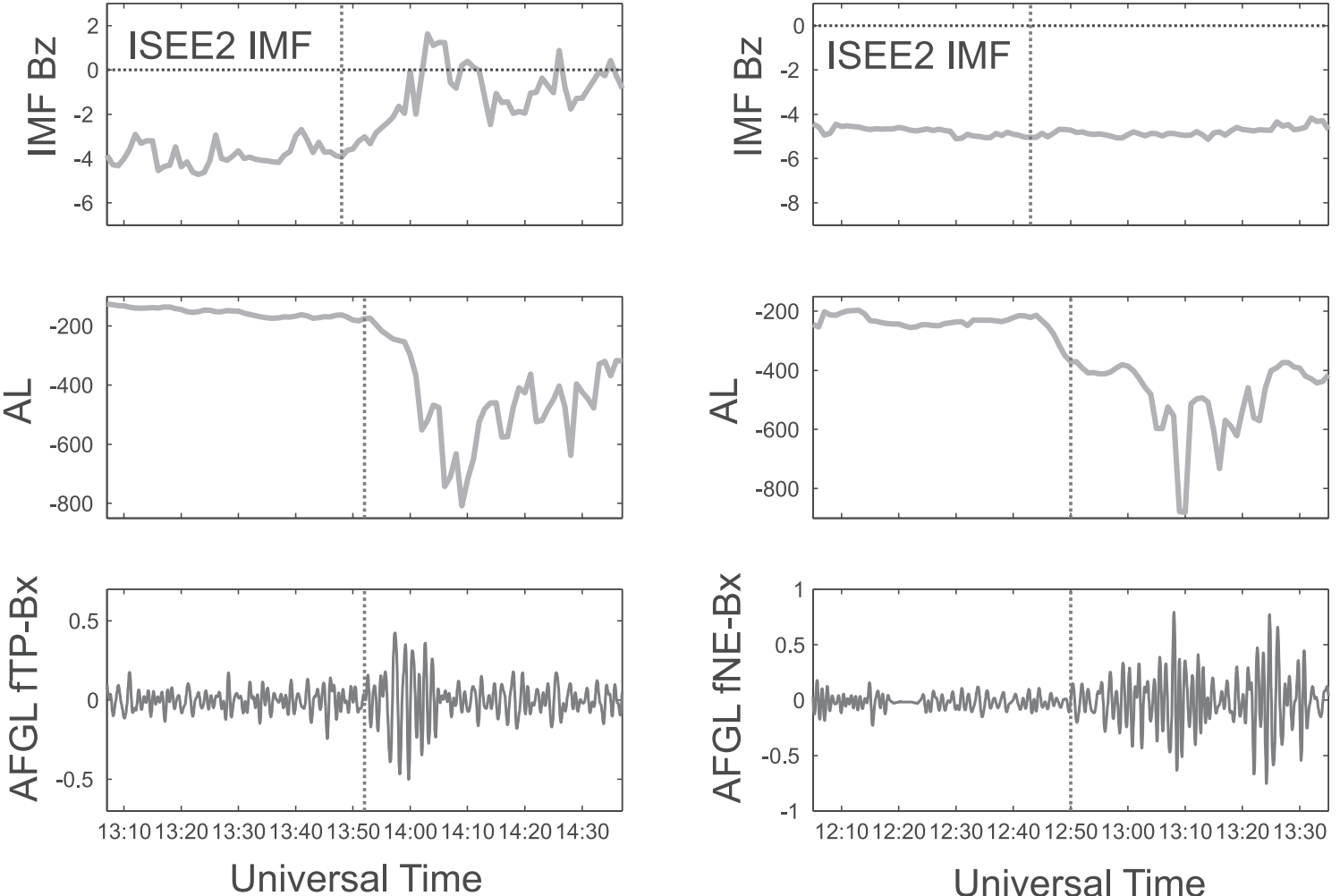

Figure 10. The ground observations for substorm onset timing are shown for comparisons. The left panels are the IMF triggered examples and the right panels are the non-IMF triggered examples. 


\section{Summary of database for March \& April in 1978,1979}

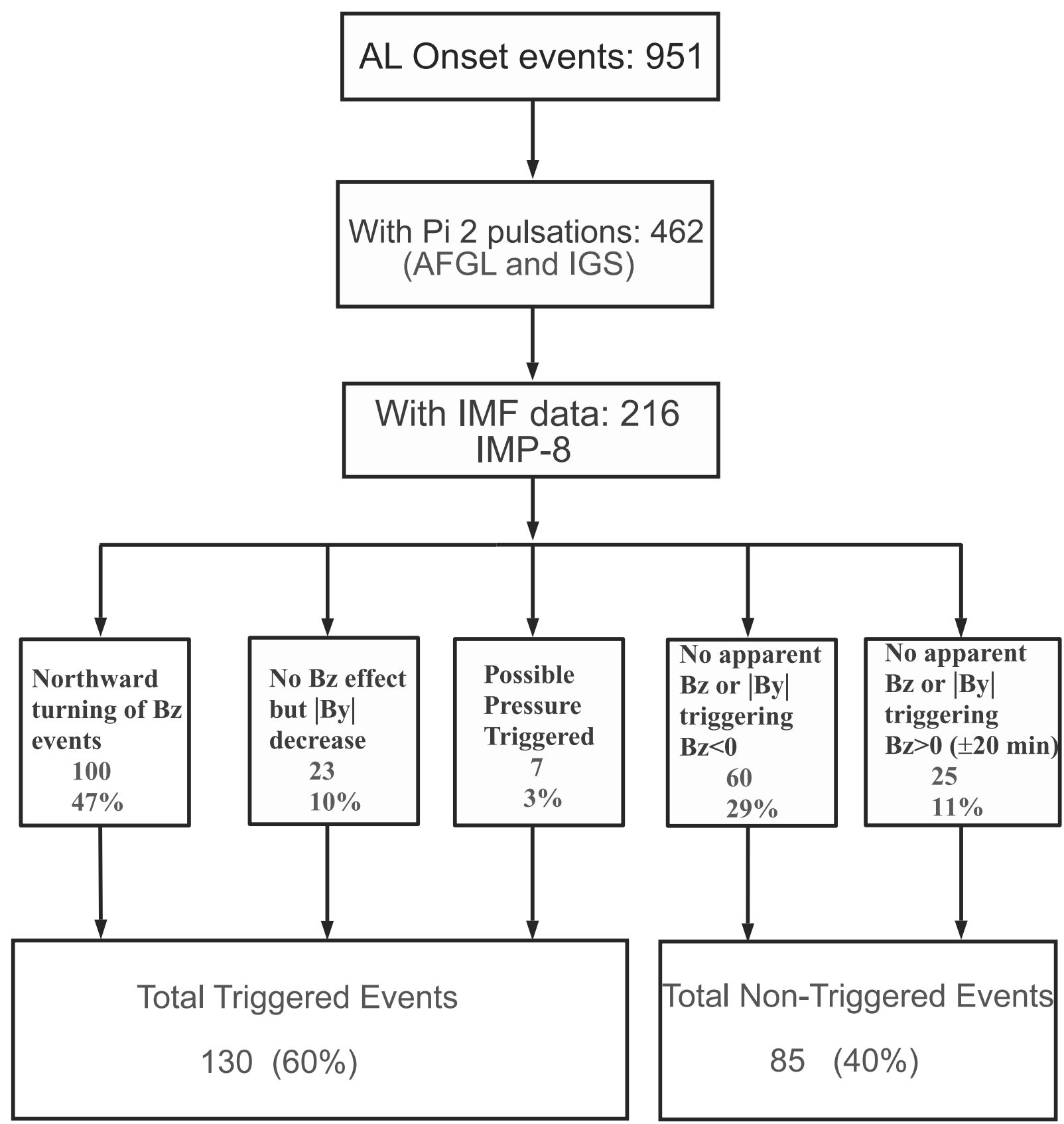

Figure 11. A summary of the aggregate statistics of triggering in the IMP-8 study. It is clear that for about $40 \%$ of the substorms no IMF triggers could be identified while for the other $60 \%$ we found either $\mathrm{B}_{\mathrm{z}}$ of $\mathrm{B}_{\mathrm{y}}$ associated perturbations.

ences in classification by the two methods. This comparison is explained in Figure 15. The lower panel presents the AL index and the solid vertical lines signify the Pi 2 onset times. The upper panel contains the IMP-8 IMF $B_{z}$ time series where the heavy circles are the times identified as possible triggers by the automatic procedure. This is clearly a multiple onset example. There are at least two events in this example. Two dashed lines corresponding to the time propagated Pi 2 onset times have been added to the upper panel. Around 0739 UT there is an IMF trigger that was identified by both procedures. However, the previous onsets at 0625 UT were identified as a triggered substorm by visual inspection but failed to satisfy the automatic procedure until some minutes later. This Pi 2 onset at 0625 UT has a prolonged growth phase but does not sustain the "jump" in magnitude long enough. Also, the short duration decreases in AL that follow this event seem to be pseudobreakups rather than a main substorm onset. It should be noticed that 


\section{Are we missing the IMF trigger?}

\section{Spatial distribution of triggered and non-triggered events}

rho is the distance from IMP 8 to earth-sun line

rho $=\operatorname{sqrt}\left(Y_{g s m}^{\wedge} 2+Z^{\prime g s m}{ }^{\wedge} 2\right)$

Time of IMP8 in each rho bin

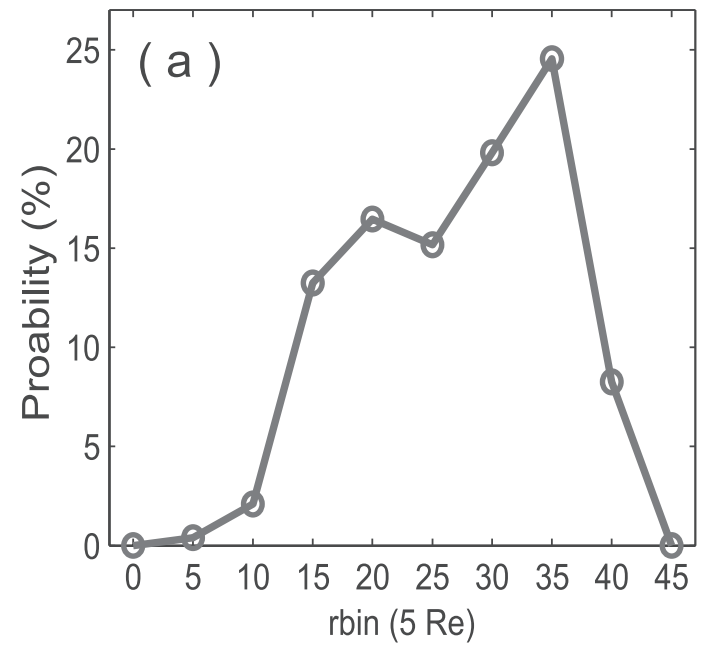

Time normalized Ratio

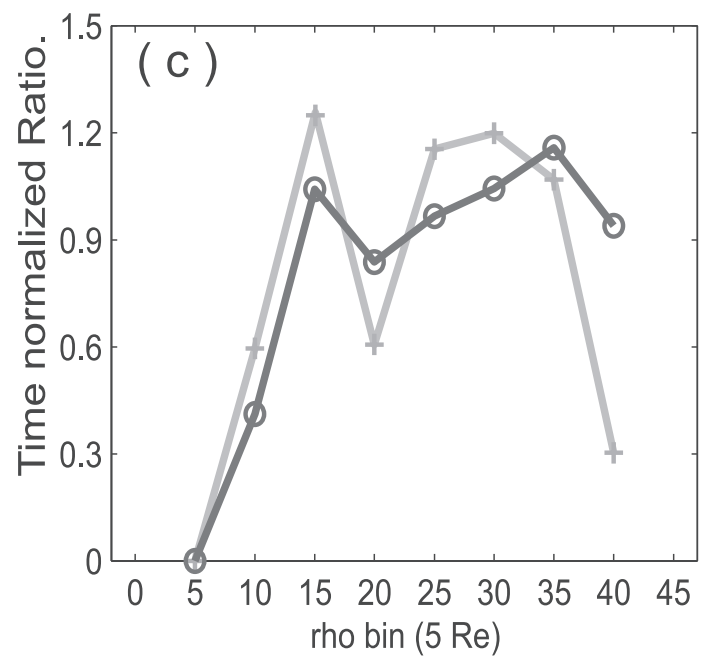

Histogram for Triggered and non-triggered events
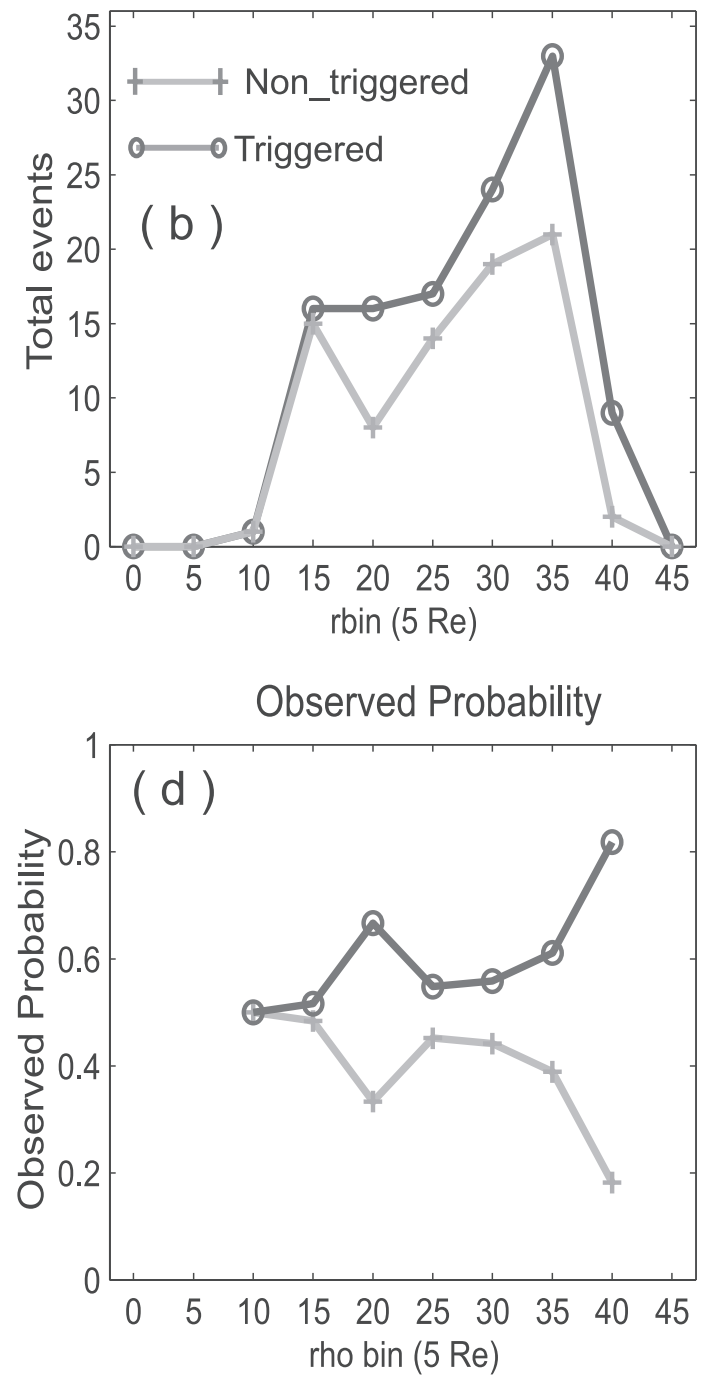

Figure 12. The spatial distribution of triggered and nontriggered events. (a) The probability that the IMP-8 satellite was in a specific bin of perpendicular distance from the Earth-Sun line. (b) The number of events of each type observed in bins at different distances from the Earth-Sun line. (c) The distance dependence of the time-normalized ratio (see text) for both classes. The results imply that the probability of observing an event of either type is directly proportional to the time spent in a bin and that the constant of proportionality is not a function of distance. (d) The observed probability of observing the two classes as a function of distance. Approximately, the occurrence probability is independent of location. The significant difference at $40 \mathrm{R}_{\mathrm{e}}$ is probably due to rare sampling and may not be meaningful.

about 20 min later an auto-identified IMF trigger occurs. Because this auto-identified trigger was more than $10 \mathrm{~min}$ after the Pi 2 onset, this event was not classified as an autoidentified trigger event. Thus there are differences between these two identification procedures. As to the other possible trigger at about 1030 UT, we cannot find the expected onset signatures (Pi 2 pulsations and sudden AL break up). Note however that AL briefly decreases after the identified trigger. 


\section{Summary of database for Sep. \& Oct. in 1978,1979}

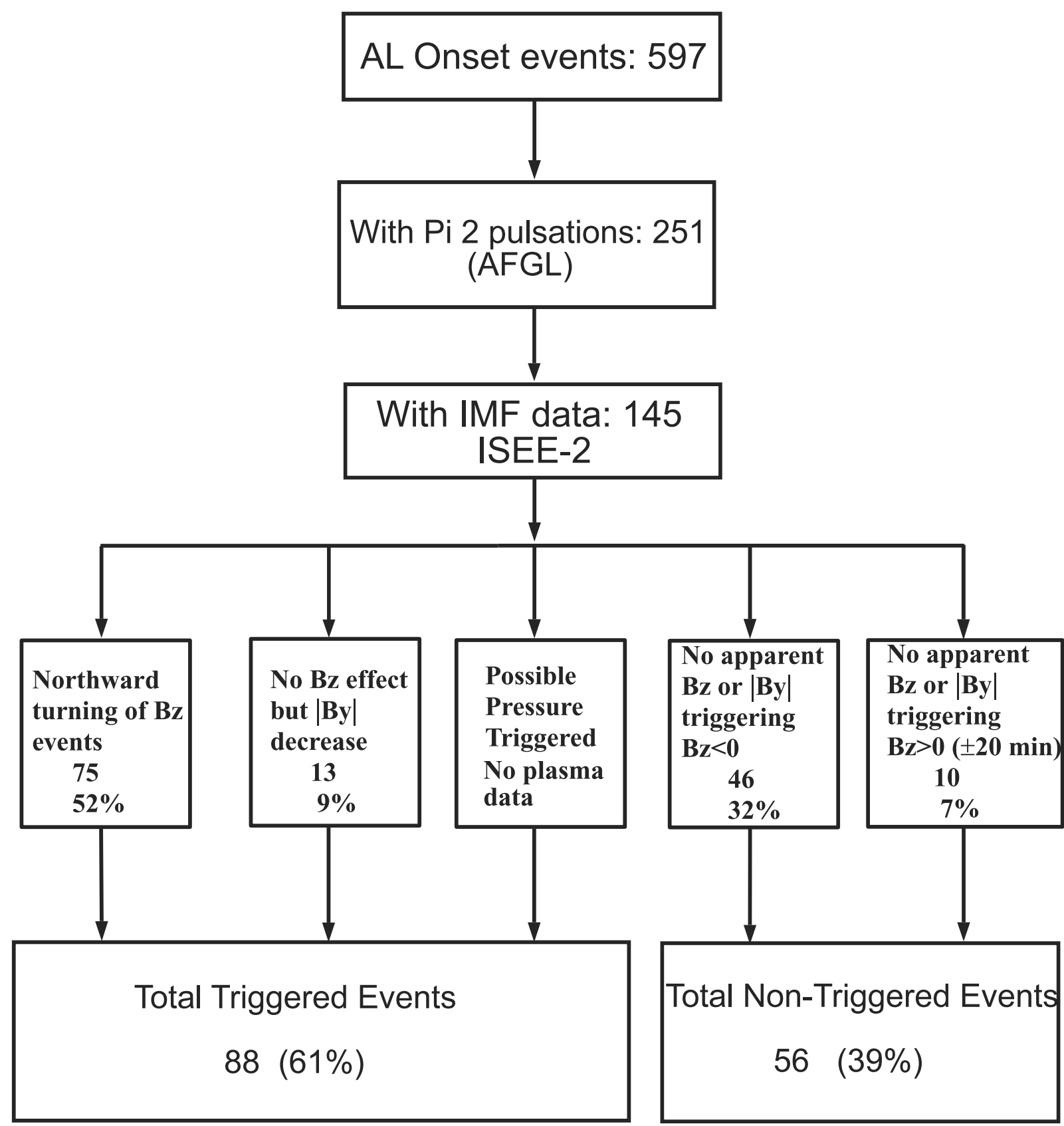

Figure 13. A summary of the aggregate statistics of triggering in the ISEE- 2 study. There are $60 \%$ of the events had either $B_{z}$ of $B_{y}$ perturbations.

[48] A comparison of the number of substorms identified by the two procedures applied to the same data set is presented in Table 3. The number of visually identified triggered substorms was 100 , of which 60 were also identified by the automatic procedure. This suggests that this automatic procedure can choose $60 \%$ of the visually selected triggers. A statistical summary is given below.

[49] Several reasons may explain the smaller percentage of substorms identified as triggered by the automatic procedure. First, the automatic criteria are more rigorous than our visual criteria. In our visual analysis we can easily compensate for small problems in the plotted data. The criteria of the automatic procedure do not take account of all of the possible waveforms of $\mathrm{Bz}$ that might trigger substorms. Second, visually we allow a jump of IMF $B_{z}$ within $4 \sim 5 \mathrm{~min}$ to be an IMF $\mathrm{B}_{\mathrm{z}}$ trigger. However, in the automatic selection a 10-min lifetime of jump was required. Finally, visually we did not specify a growth phase rigorously (IMF $\mathrm{B}_{\mathrm{z}}<0$ ), where the automatic procedure required at least a 20 -min growth phase. Finally, we found that 
Table 2. Fraction of the Substorm Onsets Apparently Triggered by a Particular Type of Change in IMF $B_{z}$ or $B_{y}$ Observed by Both IMP-8 and ISEE Spacecraft

\begin{tabular}{lcc}
\hline Satellite Name & Number, $\%$ at IMP-8 & Number, $\%$ at ISEE-2 \\
\hline Total substorm events with IMF data & $215,100 \%$ & $145,100 \%$ \\
Events possibly triggered by IMF $B_{z}$ & $100,47 \%$ & $75,52 \%$ \\
Events possibly triggered by IMF $\left|\mathrm{B}_{\mathrm{y}}\right|$ & $23,10 \%$ & $13,9 \%$ \\
Dynamic pressure triggered events & $7,3 \%$ & NA \\
Nontriggered events (IMF $\mathrm{B}_{\mathrm{z}}<0$ ) & $60,29 \%$ & $46,32 \%$ \\
Nontriggered events (IMF $\mathrm{B}_{\mathrm{z}}>0, \pm 20$ minutes around onsets) & $25,11 \%$ & $10,7 \%$ \\
\hline
\end{tabular}

IMP-8 has frequent data gaps, which frequently cause failure of the automatic procedure. All of these reduce the number of potential IMF $B_{z}$ triggers identified. This suggests that improvements in the criteria are needed.

\subsubsection{ISEE-2 Satellite}

[50] We have also applied both procedures to the ISEE-2 dataset. We found 367 possible IMF $B_{z}$ triggers in the ISEE-2 IMF observations. This number of auto-identified IMF triggers is much larger than the 145 main Pi 2 onsets identified in the same interval. The statistics are listed in Table 4.
[51] In this study the automatically identified IMF triggers correspond to about $80 \%$ of the visually identified IMF triggers. Compared with $60 \%$ for the IMP- 8 IMF observation, this is a dramatic increase. After examining the events, we found that the increase in the number of automatically identified IMF triggers is probably due to the better instrumentation of the ISEE-2 magnetometer. In the previous section we attributed the difference between the numbers of automatically and visually identified IMF triggers to data gaps. There are many fewer gaps in the ISEE-2 data, and the

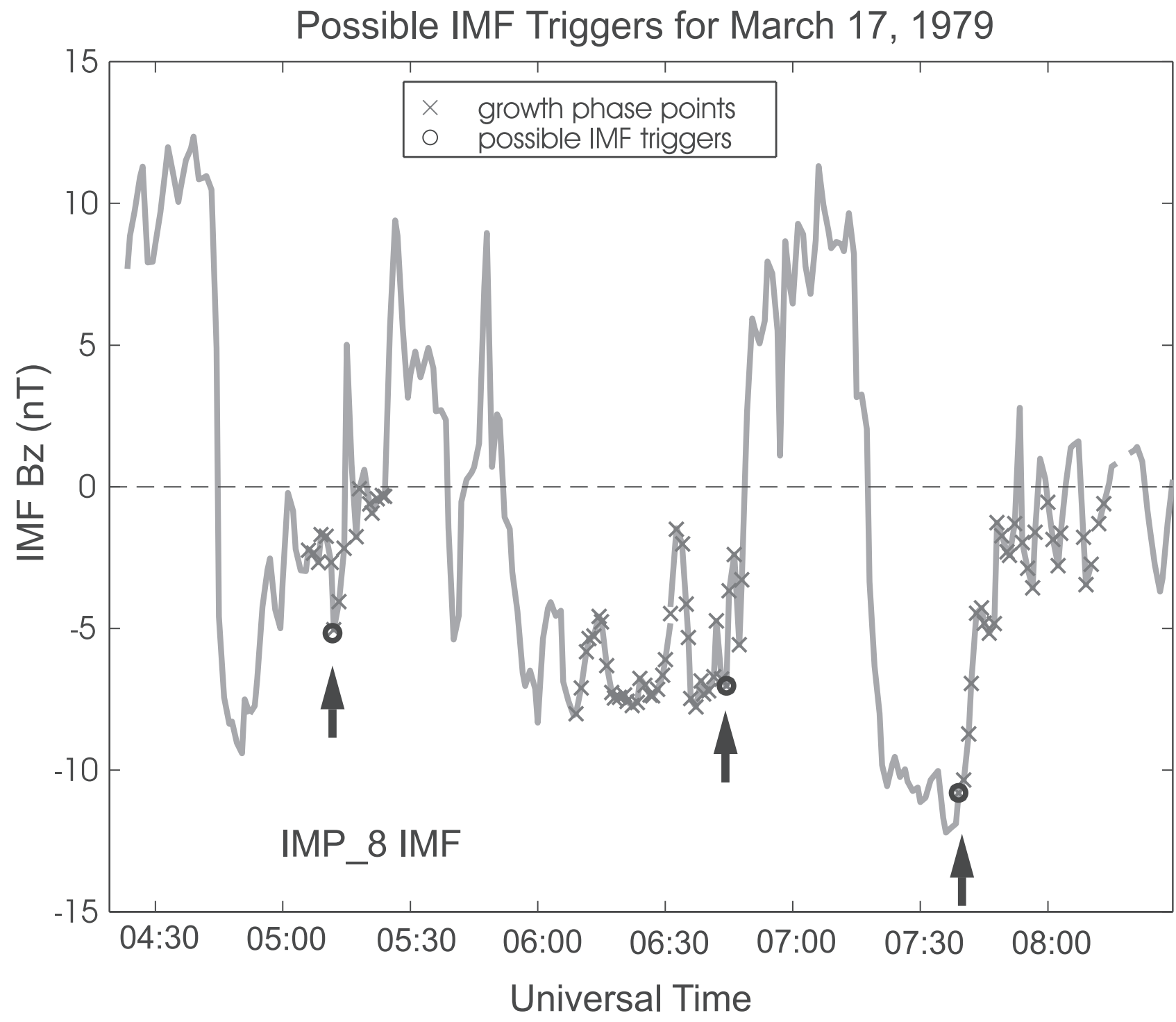

Figure 14. An example for the automatic identification of IMF $B_{z}$ triggers for IMP-8. Three possible $\mathrm{IMF} \mathrm{B}_{\mathrm{z}}$ triggers were identified by the procedure. 

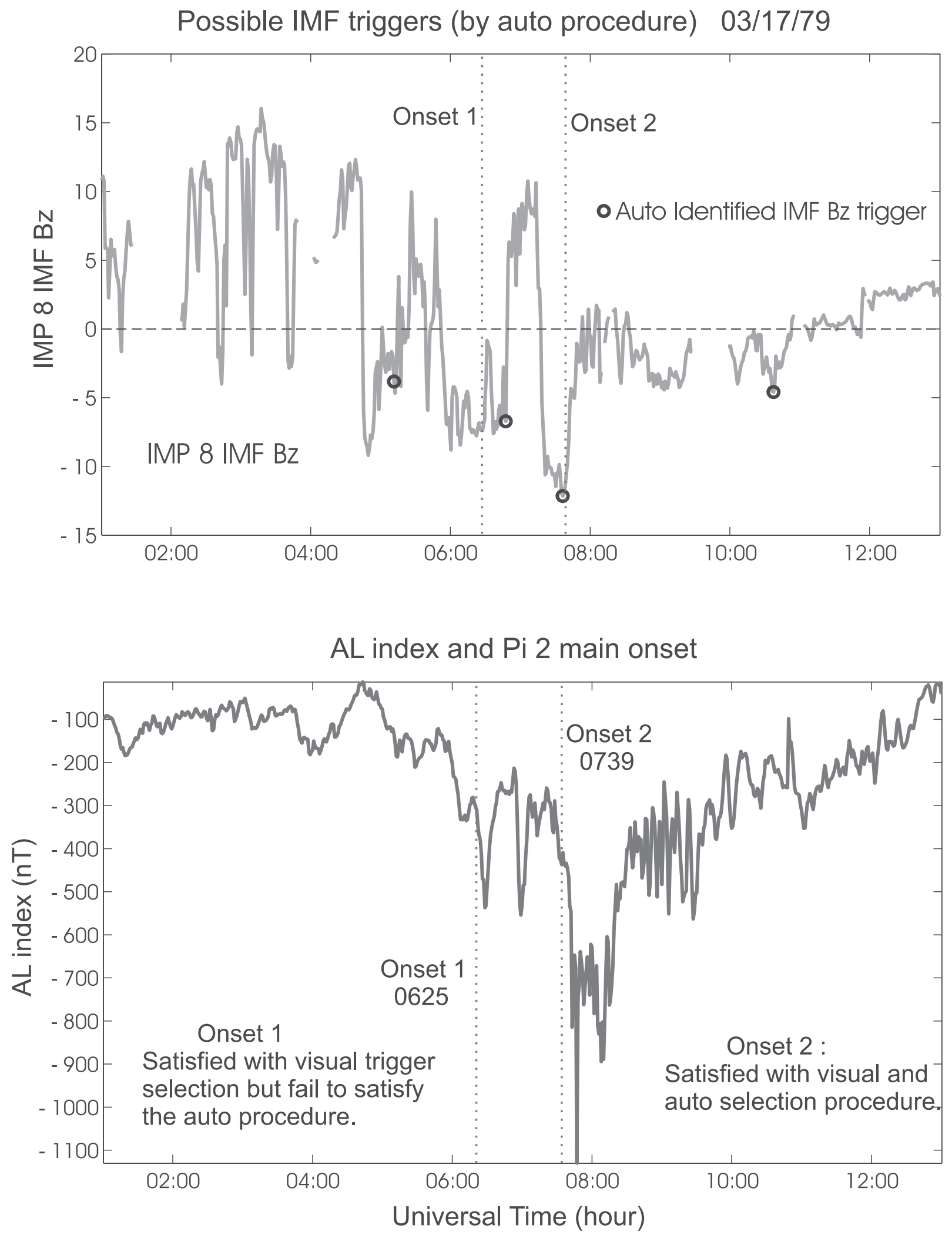

Figure 15. A comparison of the association between automatically selected $I M F B_{z}$ triggers and visually selected triggers with substorm onsets. In this example we found that one of the automatically selected $\mathrm{B}_{\mathrm{z}}$ triggers was associated with a substorm onset. The other two onsets were chosen as triggered events through visual examination but failed to satisfy the criteria of the automatic procedure. Another auto-selected $B_{z}$ trigger $(\sim 1100 \mathrm{UT})$ is associated with a slight dip in AL during the recovery phase. 
Table 3. Comparison of the Number of Triggered Substorms Identified by Visual and Automatic Analysis in the IMP-8 Data Set \begin{tabular}{ll}
\hline Procedure & Number, $\%$ \\
\hline
\end{tabular} Total substorms with IMF (IMP-8) data $215,100 \%$ Visually identified triggered substorms Automatically identified triggered substorms $60,29 \%$

correspondence between the two procedures is much better. Note, however, that in both studies the fraction of substorms that appear to be triggered on a basis of visual analysis is nearly the same, about $50 \%$. The nearly identical results for IMP-8 at all positions in its orbit, and for ISEE-2 immediately upstream of the subsolar bow shock argues that there is little difference in the probability of detecting triggers wherever IMP-8 is in its orbit. This result therefore agrees with our earlier study of the dependence of IMP-8 triggering on distance from the Earth-Sun line.

\subsection{Summary of Observations}

[52] In this study, we found the following results: (1) more than half of all substorms appear to be triggered by northward turnings, (2) $\left|\mathrm{B}_{\mathrm{y}}\right|$ changes in the absence of $\mathrm{Bz}$ increases trigger probability at most by about $10 \%$ of substorms, (3) dynamic pressure changes with no $\mathrm{Bz}$ or By changes are associated with only $3 \%$ of substorm onsets, (4) a substantial fraction of all substorm onsets $(\sim 11 \%)$ occur during prolonged northward IMF when there is no possibility of a reduction in tail electric field, (5) the probability of observing a triggered or nontriggered substorm is not a significant function of distance from the Earth-Sun line beyond $10 \mathrm{Re}$ in the IMP-8 dataset, (6) a study of triggering with ISEE-2 upstream of the bow shock and close to the Earth-Sun line obtains the same aggregate statistical results as the IMP-8 study further away, and (7) a computer algorithm designed by Lyons et al. [1997] to detect possible triggers catches only $60 \%$ of the visually identified triggers in IMP- 8 but $80 \%$ of the triggers found visually in the ISEE-2 data.

\subsection{Discussion and Conclusions}

[53] Using AE indices alone is not a very accurate way to determine substorm onsets. In fact, some of the earlier studies [Horwitz, 1985; McPherron et al., 1986] have been criticized for their timing of substorm onsets because they use only the AL index. With the help of $\mathrm{Pi} 2$ pulsations we combined these two substorm onset indicators to determine the main onset of substorms with greater accuracy. The statistical results suggest that this procedure is better than using the AL index alone. In the absence of satellite auroral images our procedure is probably the best that can be done in large statistical studies.

[54] Using this timing procedure, we have determined hundreds of substorm events, which can be used to examine

Table 4. Comparison of the Number of Triggered Substorms Identified by Visual and Automatic Analysis in the ISEE-2 Data Set

\begin{tabular}{lr}
\hline Procedure & Number, $\%$ \\
\hline Total substorms with IMF (ISEE-2) data & $145,100 \%$ \\
Visually identified triggered substorms & $75,52 \%$ \\
Automatically identified triggered substorms & $59,41 \%$ \\
\hline
\end{tabular}

the triggering hypothesis. We found that northward turning of the IMF $B_{z}$ is apparently the most important cause of substorm triggering. About $60 \%$ of the substorm onsets can be associated with a northward turning of IMF $B_{z}$. Other possible external triggers, IMF $B_{y}$, and dynamic pressure changes are not as important as $\mathrm{B}_{\mathrm{z}}$. In our analysis only about $10 \%$ of the substorms appear to be associated with a reduction of the IMF $\left|\mathrm{B}_{\mathrm{y}}\right|$ and no change in Bz. About $3 \%$ of the events appear to be triggered by dynamic pressure in the absence of either Bz or By changes. This suggests that the effects of IMF $\left|\mathrm{B}_{\mathrm{y}}\right|$ and pressure ignored in many studies cannot account for the $40 \%$ of substorms that appear to be nontriggered.

[55] Lyons et al. [1997] also suggested that the distance from the Earth-Sun line is another critical parameter in determining whether a possible IMF trigger is detected. Using observations from two satellites (IMP-8 and ISEE-2), we were unable to find any evidence supporting this hypothesis. As a matter of fact, the probability for triggered and nontriggered substorms is about the same regardless of the distance to the Earth-Sun line. The fact that there is no dramatic change with distance from the Earth-Sun line suggests that if there are small-scale structures in the solar wind, they do not have to hit the subsolar magnetopause to cause triggering. Alternatively, there really is a class of substorms that are initiated by processes internal to the magnetosphere and do not require an external trigger.

[56] An interesting result found in our study is that $10 \%$ of the substorm onsets were initiated during purely northward IMF conditions. Others studies have noted this as well. Table 5 compares the percentages found in these earlier studies.

[57] Roughly, there is about a $7 \sim 10 \%$ occurrence rate of substorms during northward field in every study. The existence of these nontriggered substorms during positive IMF $B_{z}$ suggests that a decrease of interplanetary electric field across the magnetosphere may not be necessary for substorm onset since in these cases the IMF had turned northward at least $20 \mathrm{~min}$. before expansion onset.

[58] Since the visual inspection of IMF triggering is very subjective, we have used an automatic procedure to pick IMF triggers and then examine whether the result is consistent with our visual inspection. A computer algorithm designed by Lyons et al. [1997] was used to detect possible triggers. We found that this procedure catches only $60 \%$ of

Table 5. Percentage of Substorms That Were Observed During Northward IMF Conditions When an Increase in Bz Cannot Decrease the Magnetospheric Electric Field

\begin{tabular}{lc}
\hline Referred Studies & Percentage of Substorms Under Steady Northward IMF \\
\hline McPherron et al., [1986] & $11 \%(15 / 128)$ \\
Henderson et al. $[1996]$ & $14 \%(1 / 7)$ \\
Lyons et al. $[1997]$ & $5 \%(1 / 20)$ \\
Triggering studies for spring period, 1978, 1979 (IMP-8) & $11 \%(25 / 216)$ \\
Triggering studies for fall period, 1978, 1979 (ISEE-2) & $7 \%(9 / 125)$ \\
\hline
\end{tabular}


the visually identified triggers in IMP- 8 but $80 \%$ of the triggers found visually in the ISEE-2 data. A very likely explanation for this discrepancy is the data quality. IMP 8 solar wind observations have more data gaps than ISEE-2 solar wind observations. This would suggest that our visual inspection procedure can identify most of the IMF triggers because the match up percentage between auto and visual procedures can be higher than $80 \%$ if the data are of high quality.

[59] In summary it appears that about $60 \%$ of all substorms are triggered by IMF or dynamic pressure perturbations, but about $40 \%$ of all substorms are not. This implies that some substorms may be due to internal processes in the magnetosphere but that it is very likely that this process is susceptible to external perturbations.

[60] Acknowledgments. The authors would like to thank L.R. Lyons and our colleagues at UCLA for their helpful suggestions and discussions in preparing this work. This research was funded by NSF ATM 98-19935, NSF ATM 99-72069, and NASA NAG 5-11898. Also, the California Space Institute has supported this work through grant CS-31-98.

[61] Lou-Chuang Lee thanks James L. Horwitz for the assistance in evaluating this paper.

\section{References}

Baker, D. N., R. D. Zwickl, S. J. Bame, E. W. J. Hone, B. T. Tsurutani, E. J. Smith, and S.-I. Akasofu, An ISEE 3 High Time Resolution Study of Interplanetary Parameter Correlations With Magnetospheric Activity, J. Geophys. Res., 88(A8), 6230-6242, 1983.

Blanchard, G. T., L. R. Lyons, and J. Spann, Predictions of substorms following northward turnings of the interplanetary magnetic field, J. Geophys. Res., 105(A1), 375-384, 2000.

Burch, J. L., Triggering of the substorm expansion phase by directional discontinuities in the interplanetary magnetic field, Eos Trans. AGU, 54, 413, 1973.

Caan, M. N., R. L. McPherron, and C. T. Russell, Substorm and interplanetary magnetic field effects on the geomagnetic tail lobes, J. Geophys. Res., 80(1), 191-194, 1975.

Caan, M. N., R. L. McPherron, and C. T. Russell, Characteristics of the association between the interplanetary magnetic field and substorms, J. Geophys. Res., 82(29), 4837-4842, 1977.

Coroniti, F. V., Explosive tail reconnection: The growth and expansion phase of magnetospheric substorms, J. Geophys. Res., 90(A8), $7427-$ 7447, 1985

Dmitrieva, N. P., and V. A. Sergeev, The spontaneous and induced onset of the explosive phase of a magnetospheric substorm and the duration of its preliminary phase, Geomagn. Aeron., 23(3), 380-383, 1983.

Henderson, M. G., G. D. Reeves, R. D. Belian, and J. S. Murphree, Observation of magnetospheric substorms occurring with no apparent solar wind/IMF trigger, J. Geophys. Res., 101(A5), 10,773-10,791, 1996.

Hones, E. W., Jr., J. R. Asbridge, S. J. Bame, and S. Singer, Substorm variations of the magnetotail plasma sheet from Xsm $=-6 \mathrm{Re}$ to Xsm $=$ -60 Re, J. Geophys. Res., 78(1), 109-132, 1973.

Horwitz, J. L., The substorm as an internal magnetospheric instability: Substorms and their characteristic time scales during intervals of steady interplanetary magnetic field, J. Geophys. Res., 90(A5), 4164-4170, 1985.

Hsu, T.-S., and R. L. McPherron, Occurrence frequency of substorm field and plasma signatures observed near-earth by ISEE-1/2, in Proceedings of Third International Conference on Substorms (ICS-3), edited by E. J. Rolfe and B. Kaldeich, pp. 333-340, Eur. Space Agency, Versailles, France, 1996
Hsu, T.-S., and R. L. McPherron, The main onset of a magnetospheric substorm, in Proceedings of Fourth International Conference on Substorms (ICS-4), edited by S. Kokubun and Y. Kamide, pp. 79-82, Terra Sci., Tokyo, 1998.

Hsu, T.-S., and R. L. McPherron, An evaluation of the statistical significance of the association between northward turnings of the IMF and substorm expansion onsets, J. Geophys. Res., 107(A11), 1398, doi:10.1029/2000JA000125, 2002.

Iijima, T., and T. Nagata, Signatures for substorm development of the growth phase and expansion phase, Planet. Space Sci., 20(7), 10951112, 1972

Knecht, D. J., Daily magnetometer for 1978 from the AFGL network, Tech. Rep. AFGL-TR-85-0027, 243 pp., Air Force Geophys. Lab., Hanscom Air Force Base, Mass., 1985.

Lui, A. T. Y., R. E. Lopez, S. M. Krimigis, R. W. McEntire, L. J. Zanetti, and T. A. Potemra, A case study of magnetotail current sheet disruption and diversion, Geophys. Res. Lett., 15(7), 721-724, 1988.

Lyons, L. R., A new theory for magnetospheric substorms, J. Geophys. Res., 100(A10), 19,069-19,081, 1995.

Lyons, L. R., Evidence suggests external triggering of substorms, Eos Trans. $A G U, 77(9), 88-89,1996 \mathrm{a}$.

Lyons, L. R., Substorms: Fundamental observation features, distinction from other disturbances and external triggering, J. Geophys. Res., 101(A6), 13,011-13,026, 1996b.

Lyons, L. R., G. T. Blanchard, J. C. Samson, R. P. Lepping, T. Yamamoto, and T. Moretto, Coordinated observations demonstrating external substorm triggering, J. Geophys. Res., 102(A12), 27,039-27,051, 1997.

McPherron, L. M., Midlatitute magnetometer chains during the IMS, in The IMF Source Book: Guide to the International Magnetospheric Study Data Analysis, edited by C. T. Russell and D. J. Southwood, pp. 170181, AGU, Washington, D.C., 1992.

McPherron, R. L., T. Terasawa, and A. Nishida, Solar wind triggering of substorm expansion onset, J. Geomagn. Geoelectr., 38(11), 1089-1108, 1986.

Nishida, A., Coherence of geomagnetic DP 2 fluctuations with interplanetary magnetic variations, J. Geophys. Res., 73(17), 5549-5559, 1968.

Pytte, T., R. L. McPherron, E. W. Hones Jr., and H. I. West Jr., Multiplesatellite studies of magnetospheric substorms: 3 . Distinction between polar substorms and convection-driven negative bays, J. Geophys. Res., 83(A2), 663-679, 1978.

Rostoker, G., Triggering of expansion phase intensifications of magneticspheric substorms by northward turning of the interplanetary magnetic field, J. Geophys. Res., 88, 6981, 1983.

Rostoker, G., W. Baumjohann, and C. T. Russell, A case study of the response of the magnetosphere to changes in the interplanetary medium, J. Geophys., 53, 170-181, 1983.

Roux, A., S. Perraut, P. Robert, A. Morane, A. Pedersen, A. Korth, G. Kremser, B. Aparicio, D. Rodgers, and R. Pellinen, Plasma sheet instability related to the westward traveling surge, J. Geophys. Res., 96(A10), 17,697-17,714, 1991.

Samson, J. C., and K. L. Yeung, Some generalizations on the method of superposed epoch analysis, Planet. Space Sci., 34(11), 1133-1142, 1986.

Stuart, W. F., Arrays of magnetometers operated NW Europe, in The IMF Source Book: Guide to the International Magnetospheric Study Data Analysis, edited by C. T. Russell and D. J. Southwood, pp. 141-152, AGU, Washington, D.C., 1982.

Troshichev, O. A., A. L. Kotikov, B. D. Bolotinskaya, and V. G. Andrezen, Influence of the IMF azimuthal component on magnetospheric substorm dynamics, J. Geomagn. Geoelectr., 38, 1075, 1986.

Yahnin, A., et al., Features of steady magnetospheric convection, J. Geophys. Res., 99, 4309, 1994.

T.-S. Hsu and R. L. McPherron, Institute of Geophysical and Planetary Physics, University of California, Los Angeles, 405 Hilgard Avenue, Los Angeles, CA 90095, USA. (thsu@igpp.ucla.edu; rmcpherron@igpp.ucla. edu) 